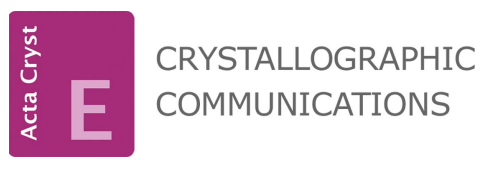

ISSN 2056-9890

Received 13 October 2016

Accepted 5 November 2016

Edited by M. Weil, Vienna University of Technology, Austria

Keywords: crystal structure; lithium; silver; amidinate; alkynylamidinate; co-crystal.

CCDC references: $1515190 ; 1515191$

Supporting information: this article has supporting information at journals.iucr.org/e

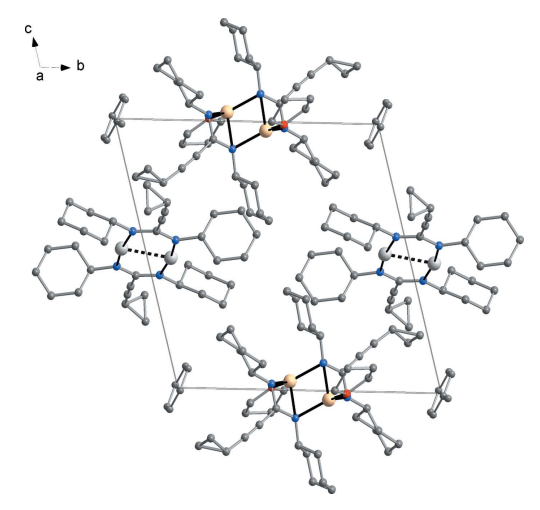

OPEN $\odot$ ACCESS

\section{Crystal and molecular structures of two silver(I) amidinates, including an unexpected co-crystal with a lithium amidinate}

\author{
Sida Wang, Nicole Harmgarth, Phil Liebing and Frank T. Edelmann*
}

Chemisches Institut der Otto-von-Guericke-Universität Magdeburg, Universitätsplatz 2, 39106 Magdeburg, Germany. *Correspondence e-mail: frank.edelmann@ovgu.de

The silver(I) amidinates bis $\left[\mu-N^{1}, N^{2}\right.$-bis(propan-2-yl)benzamidinato- $\left.\kappa^{2} N^{1}: N^{2}\right]$ disilver(I), $\left[\mathrm{Ag}_{2}\left(\mathrm{C}_{13} \mathrm{H}_{19} \mathrm{~N}_{2}\right)_{2}\right]$ or $\left[\mathrm{Ag}\left\{\operatorname{PhC}\left(\mathrm{N}^{i} \mathrm{Pr}\right)_{2}\right\}\right]_{2}(\mathbf{1})$, and bis $\left(\mu-N^{1}, N^{2}\right.$-dicyclohexyl-3-cyclopropylpropynamidinato- $\left.\kappa^{2} N^{1}: N^{2}\right)$ disilver(I), $\left[\mathrm{Ag}_{2}\left(\mathrm{C}_{18} \mathrm{H}_{27} \mathrm{~N}_{2}\right)_{2}\right]$ or $\left[\mathrm{Ag}\left\{\text { cyclo }-\mathrm{C}_{3} \mathrm{H}_{5}-\mathrm{C} \equiv \mathrm{C}-\mathrm{C}(\mathrm{NCy})_{2}\right\}\right]_{2}(\mathbf{2 a})$, exist as centrosymmetric dimers with a planar $\mathrm{Ag}_{2} \mathrm{~N}_{4} \mathrm{C}_{2}$ ring and a common linear coordination of the metal atoms in the crystalline state. Moiety 2a forms a co-crystal with the related lithium amidinate, namely bis $\left(\mu-N^{1}, N^{2}\right.$-dicyclohexyl-3-cyclopropylpropynamidinato$\left.\kappa^{2} N^{1}: N^{2}\right)$ disilver(I) $\quad$ bis $\left(\mu-N^{1}, N^{2}\right.$-dicyclohexyl-3-cyclopropylpropynamidinato$\left.\kappa^{3} N^{1}, N^{2}: N^{1}\right)$ bis(tetrahydrofuran- $\kappa O$ )lithium(I) toluene monosolvate, $\left[\mathrm{Ag}_{2}\left(\mathrm{C}_{18} \mathrm{H}_{27} \mathrm{~N}_{2}\right)_{2}\right]\left[\mathrm{Li}_{2}\left(\mathrm{C}_{18} \mathrm{H}_{27} \mathrm{~N}_{2}\right)_{2}\left(\mathrm{C}_{4} \mathrm{H}_{8} \mathrm{O}\right)_{2}\right] \cdot \mathrm{C}_{7} \mathrm{H}_{8}$ or $\quad\left[\mathrm{Ag}\left\{\right.\right.$ cyclo- $\mathrm{C}_{3} \mathrm{H}_{5}-\mathrm{C} \equiv C-$ $\left.\left.\mathrm{C}(\mathrm{NCy})_{2}\right\}\right]_{2}\left[\mathrm{Li}\left\{\text { cyclo }-\mathrm{C}_{3} \mathrm{H}_{5}-\mathrm{C} \equiv \mathrm{C}-\mathrm{C}(\mathrm{NCy})_{2}\right\}(\mathrm{THF})\right]_{2} \cdot \mathrm{C}_{7} \mathrm{H}_{8}$, composed as $\mathbf{2 a} \times$ $\mathbf{2 b} \times$ toluene. The lithium moiety $\mathbf{2 b}$ features a typical ladder-type dimeric structure with a distorted tetrahedral coordination of the metal atoms. In the silver(I) derivatives $\mathbf{1}$ and 2a, the amidinate ligand adopts a $\mu-\kappa N: \kappa N^{\prime}$ coordination, while it is a $\mu-\kappa N: \kappa N: \kappa N^{\prime}$-coordination in the case of lithium derivative $\mathbf{2 b}$.

\section{Chemical context}

Anionic $N$-chelating donor ligands such as amidinates, $\left[R \mathrm{C}(\mathrm{N} R)_{2}\right]^{-}$, and guanidinates, $\left[R_{2} \mathrm{NC}(\mathrm{N} R)_{2}\right]^{-}$, have gained tremendous importance in various fields of organometallic and coordination chemistry over the past two decades. Formally, amidinate anions are the nitrogen analogues of the carboxylate anions, while guanidinates are similarly related to the carbamates. However, in contrast to the carboxylates and carbamates, the steric properties of amidinates and guanidinates can be widely tuned through the use of different substituents, both at the outer nitrogen atoms as well as at the central carbon atom of the NCN unit. Both types of $\mathrm{N}$-chelating ligands are often regarded as 'steric cyclopentadienyl equivalents' (Bailey \& Pace, 2001; Collins, 2011; Edelmann, 2008, 2013). Meanwhile, amidinato and guanidinato complexes are known for virtually every metallic element in the Periodic Table ranging from lithium to uranium (Edelmann, 2008, 2009, 2012, 2013; Trifonov, 2010). Alkyl-substituted amidinate and guanidinate complexes of various metals have also been established as ALD and MOCVD precursors for the deposition of thin layers of metals, metal oxides, metal nitrides etc. (Devi, 2013). The most important starting materials in this field are lithium amidinates and guanidinates. Lithium amidinates are normally prepared in a straightforward manner by addition of lithium alkyls to $N, N^{\prime}$-diorganocarbodiimides in a 1:1 molar ratio, while lithium guanidinates 
are formed when lithium- $N, N$-dialkylamides are added to $N, N^{\prime}$-diorganocarbodiimides (Stalke et al., 1992; Aharonovich et al., 2008; Chlupatý et al., 2011; Nevoralová et al., 2013; Hong et al., 2013). On the other hand, silver(I) amidinates and guanidinates (Archibald et al., 2000; Lim et al., 2003; Whitehorne et al., 2011; Lane et al., 2014) are of significant importance as potential precursors for vapor deposition processes (Lim et al., 2003; Whitehorne et al., 2011), as precursors for silver nanoparticles (Cure et al., 2015), or as intermediates in silver-catalyzed amidination and guanylation reactions (Pereshivko et al., 2011; Okano et al., 2012; Li et al., 2015).

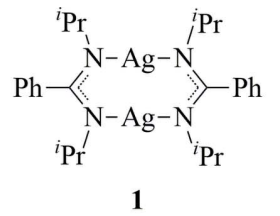

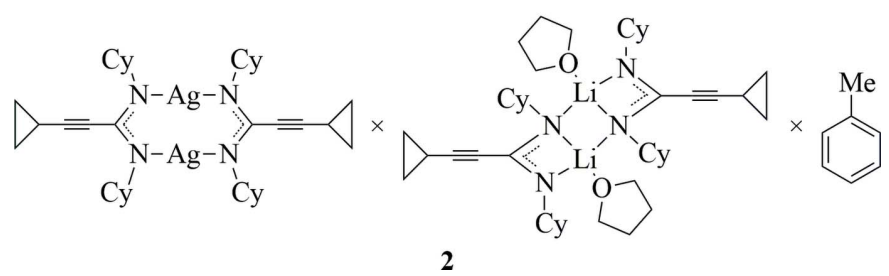

We report here the structural characterization of two silver(I) amidinates, namely $\left[\mathrm{Ag}\left\{\mathrm{PhC}\left(\mathrm{N}^{i} \mathrm{Pr}\right)_{2}\right\}\right]_{2}(\mathbf{1})$, and the unexpected co-crystal (2), composed as $\left[\mathrm{Ag}\left\{\right.\right.$ cyclo- $\mathrm{C}_{3} \mathrm{H}_{5}-$ $\left.\left.\mathrm{C} \equiv \mathrm{C}-\mathrm{C}(\mathrm{NCy})_{2}\right\}\right]_{2} \quad$ (2a) $\quad \times \quad\left[\mathrm{Li}\left\{\right.\right.$ cyclo $-\mathrm{C}_{3} \mathrm{H}_{5}-\mathrm{C} \equiv \mathrm{C}-$ $\left.\left.\mathrm{C}(\mathrm{NCy})_{2}\right\}(\mathrm{THF})\right]_{2}(\mathbf{2 b}) \times$ toluene $(\mathrm{Cy}=$ cyclohexyl $)$.

\section{Structural commentary}

Silver(I) compound 1 (Fig. 1) and silver moiety 2a (Fig. 2): Both silver(I) complexes exist as centrosymmetric dimers in the crystalline state. Compound $\mathbf{1}$ crystallizes without any solvent, and the molecular structure of moiety $\mathbf{2 a}$ was determined from the co-crystal $\mathbf{2}(\mathbf{2} \mathbf{a} \times \mathbf{2 b} \times$ toluene $)$. In both $\mathbf{1}$ and 2a, each of the two $\mathrm{N}$ atoms of the amidinate ligand coordinates to one $\mathrm{Ag}$ atom (coordination mode $\mu-\kappa N: \kappa N^{\prime}$ ), and the

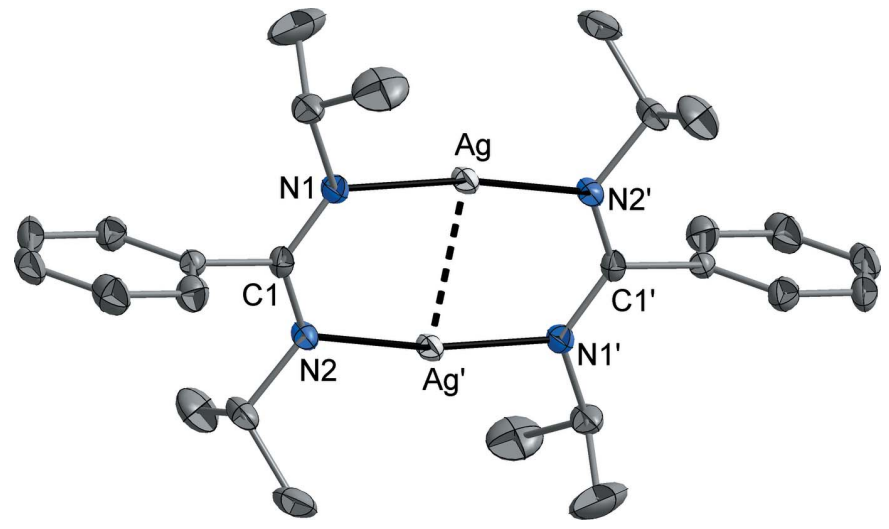

Figure 1

The molecular structure of compound 1. Displacement ellipsoids are drawn at the $50 \%$ probability level and $\mathrm{H}$ atoms have been omitted for clarity. [Symmetry code: (') $2-x, 2-y, 1-z$.]
$\mathrm{Ag}$ atoms adopt an almost linear coordination $[\mathbf{1}: \mathrm{N}-\mathrm{Ag}-\mathrm{N}$ $\left.170.58(7)^{\circ} ; 2 \mathrm{a}: \mathrm{N}-\mathrm{Ag}-\mathrm{N} 170.66(5)^{\circ}\right]$ by two $\mathrm{N}$ atoms of two symmetry-related amidinate ligands, leading to centrosymmetric dimers in each case. The $\mathrm{Ag}-\mathrm{N}$ separations are very similar in both structures [1: 2.0959 (16) and 2.0965 (16) $\AA$, 2a: 2.0908 (15) and 2.0916 (14) $\AA$ ]. An $s p^{2}$ hybridization can be assigned to the $\mathrm{N}$ atoms since the coordination environment is almost trigonal-planar. The $\mathrm{C}-\mathrm{N}$ separations within the amidinate NCN fragment are virtually equal [1: twice 1.322 (3) $\AA$, 2a: 1.329 (2) and 1.331 (2) $\AA$ ], indicating a typical delocalization of the negative charge. Through the mentioned connectivity pattern, a strictly planar $\mathrm{C}_{2} \mathrm{~N}_{4} \mathrm{Ag}_{2}$ eightmembered ring with a short $\mathrm{Ag} \cdots \mathrm{Ag}$ contact is built $[\mathbf{1}$ : 2.6604 (3) $\AA$, 2a: 2.6838 (3) $\mathrm{A}]$. This constitution might be supported by some attractive $d^{10}-d^{10}$ interaction between the $\mathrm{Ag}$ atoms that have been frequently discussed in the literature (for a review, e.g. see: Jansen, 1987). The molecular structures of the here discussed compounds are closely related to those of the most previously described copper(I) and silver(I) amidinates, namely $\left[\mathrm{Cu}_{2}\left\{R \mathrm{C}\left(\mathrm{N} R^{\prime}\right)_{2}\right\}_{2}\right]\left(R, R^{\prime}=\mathrm{Me},{ }^{n} \mathrm{Bu} ; \mathrm{Li}\right.$ et al., 2005) and $\left[M_{2}\left\{\mathrm{MeC}\left(\mathrm{N}^{i} \mathrm{Pr}\right)_{2}\right\}_{2}\right](M=\mathrm{Cu}, \mathrm{Ag})$. However, in the case of $\mathrm{Ag}\left\{\mathrm{MeC}\left(\mathrm{N}^{i} \mathrm{Pr}\right)_{2}\right\}$, also a trimeric structure $\left[\mathrm{Ag}_{3}\left\{\mathrm{MeC}\left(\mathrm{N}^{i} \mathrm{Pr}\right)_{2}\right\}_{3}\right]$ was observed (Lim et al., 2003). The bond lengths and angles involving the $\mathrm{Ag}$ atoms, $v i z . \mathrm{Ag}-\mathrm{N}$ and $\mathrm{Ag}-\mathrm{Ag}$ distances and $\mathrm{N}-\mathrm{Ag}-\mathrm{N}$ angles, in the compounds discussed herein resemble those observed in the previously reported dimeric silver(I) amidinates. A dimerization or oligomerization under formation of linear $\mathrm{N}-M-\mathrm{N}$ units is also typical for a broad ensemble of copper(I) and silver(I) complexes with other anionic nitrogen ligands, e.g. $\left[\mathrm{Cu}_{4}\left(\mathrm{~N} R_{2}\right)_{4}\right]$ (e.g. $R=\mathrm{Me}$, Gambarotta et al., $1987 ; R=\mathrm{Et}$, Hope \& Power, 1984; $R=\mathrm{SiMe}_{3}$, James et al., 1998), $\left[\mathrm{Ag}_{4}\left(\mathrm{~N}\left(\mathrm{SiMe}_{3}\right)_{2}\right\}_{4}\right]$ and $\left[\mathrm{Ag}_{3}\left(N, N, N^{\prime}, N^{\prime}\right.\right.$-tetramethylpiperid$\left.\mathrm{yl})_{3}\right]$ (Hitchcock et al., 1996), $\left[\mathrm{Cu}_{2} \mathrm{Tl}_{2}(\text { ThioSila) })_{2}\right]$ and $\left[\mathrm{Ag}_{4}(\text { ThioSila })_{2}(\mathrm{THT})_{2}\right] \quad\left(\right.$ ThioSila $=\left\{\mathrm{Me}_{2} \mathrm{Si}\left(\mathrm{N}-\mathrm{C}_{6} \mathrm{H}_{4}-2-\right.\right.$ $\left.\mathrm{SPh})_{2}\right\}^{2-}$, THT = tetrahydrothiophene; Liebing \& Merzweiler,

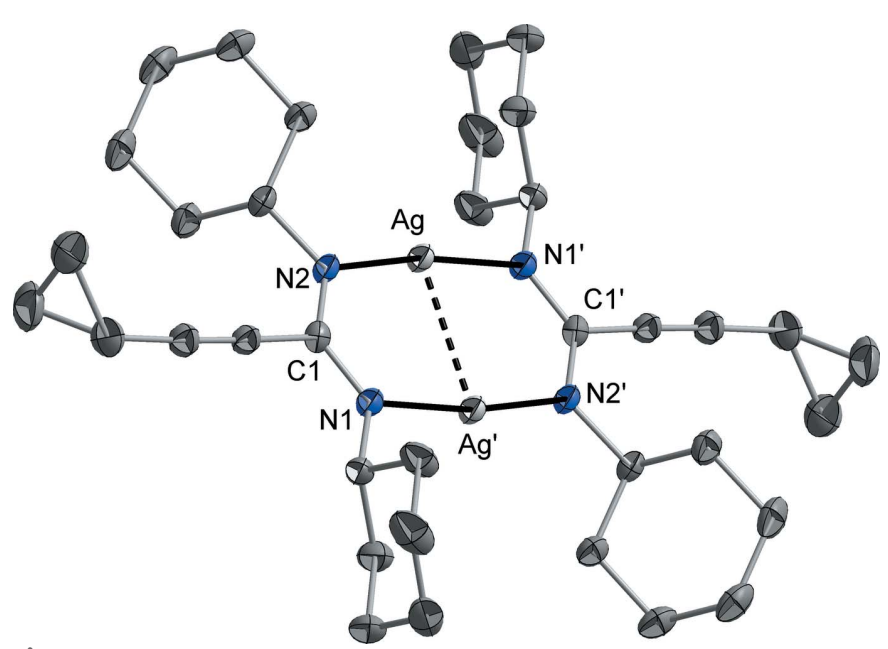

Figure 2

The molecular structure of moiety $\mathbf{2 a}$, determined from the co-crystal $\mathbf{2}$. Displacement ellipsoids are drawn at the $50 \%$ probability level and $\mathrm{H}$ atoms have been omitted for clarity. [Symmetry code: $\left({ }^{\prime}\right) 2-x, 2-y$, $1-z$.] 


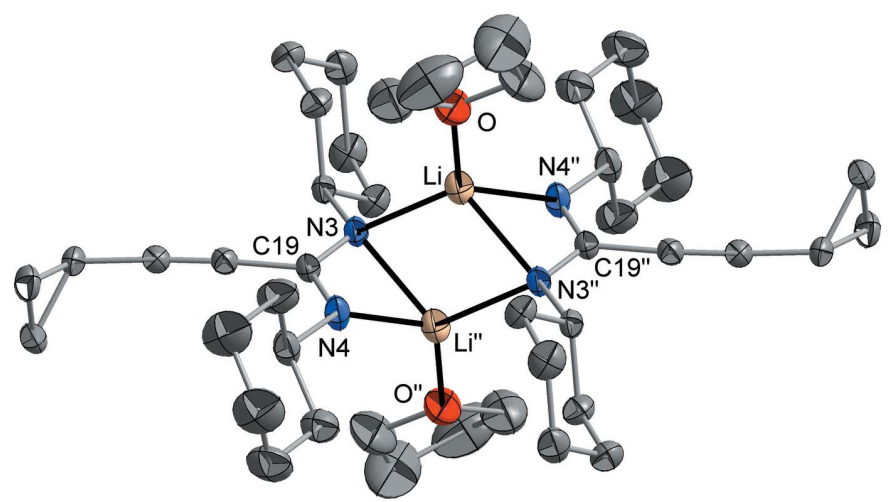

Figure 3

The molecular structure of moiety $\mathbf{2 a}$, determined from the co-crystal $\mathbf{2}$. Displacement ellipsoids are drawn at the $50 \%$ probability level and $\mathrm{H}$ atoms have been omitted for clarity. [Symmetry code: (") $2-x, 1-y$, $-z$.]

2016). The silane diamide complexes $\left[M_{4}(\text { ThioSila })_{2}\right]$ comprise a planar $\mathrm{Si}_{2} \mathrm{~N}_{4} M_{2}$ ring that is structurally closely related to the $\mathrm{C}_{2} \mathrm{~N}_{4} M_{2}$ ring in the dimeric amidinate complexes.

Lithium moiety $\mathbf{2 b}$ (Fig. 3): The molecular structure of $\mathbf{2 b}$ was determined from the above-mentioned co-crystal $2(\mathbf{2 a} \times$ $\mathbf{2 b} \times$ toluene). Like the silver components $\mathbf{1}$ and 2a, the lithium moiety exists as a centrosymmetric dimer in the crystalline state. However, the molecular structure of $\mathbf{2 b}$ is considerably different, featuring a centrosymmetric $\mathrm{Li}_{2} \mathrm{~N}_{2}$ four-membered ring formed by $\mu$-bridging coordination of one of the $\mathrm{N}$ atoms $(\mathrm{N} 3)$. The $\mathrm{Li}-\mathrm{N}$ distances within this ring are 2.033 (4)-2.261 (4) $\AA$ and therefore in the expected range. The coordination number of the mentioned $\mathrm{N}$ atom N3 is consequently raised to four and an $s p^{3}$ hybridization fits best to describe the bonding situation. The second $\mathrm{N}$ atom of the amidinate ligand (N4) is attached to only one $\mathrm{Li}$ atom with a shorter $\mathrm{Li}-\mathrm{N}$ bond of 2.001 (4) $\AA$, and its coordination environment is trigonal-planar like in the related silver

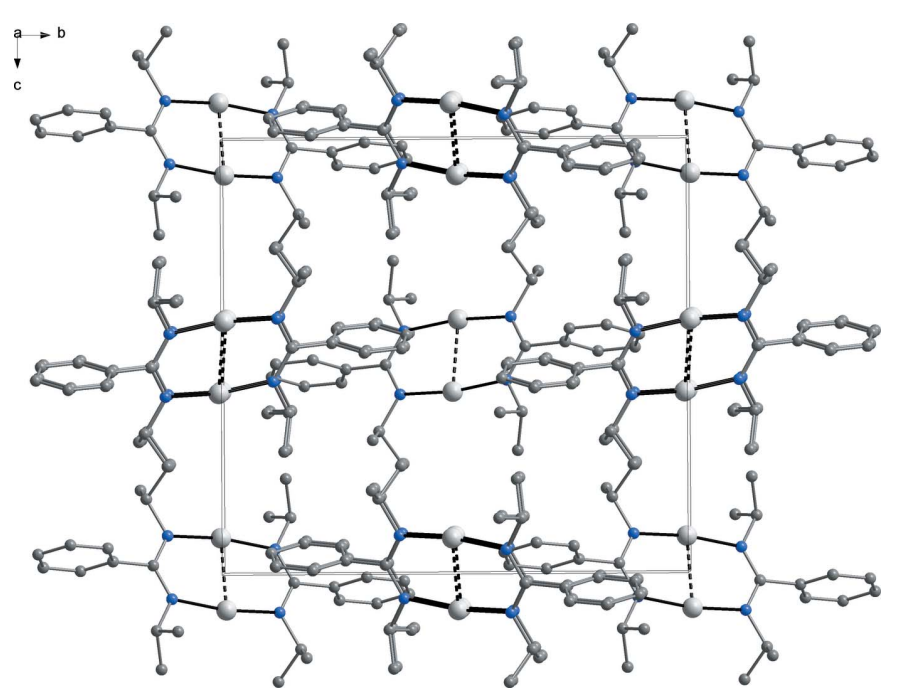

Figure 4

Crystal packing of dimeric silver(I) amidinate molecules in compound $\mathbf{1}$, viewed in a projection on (100).

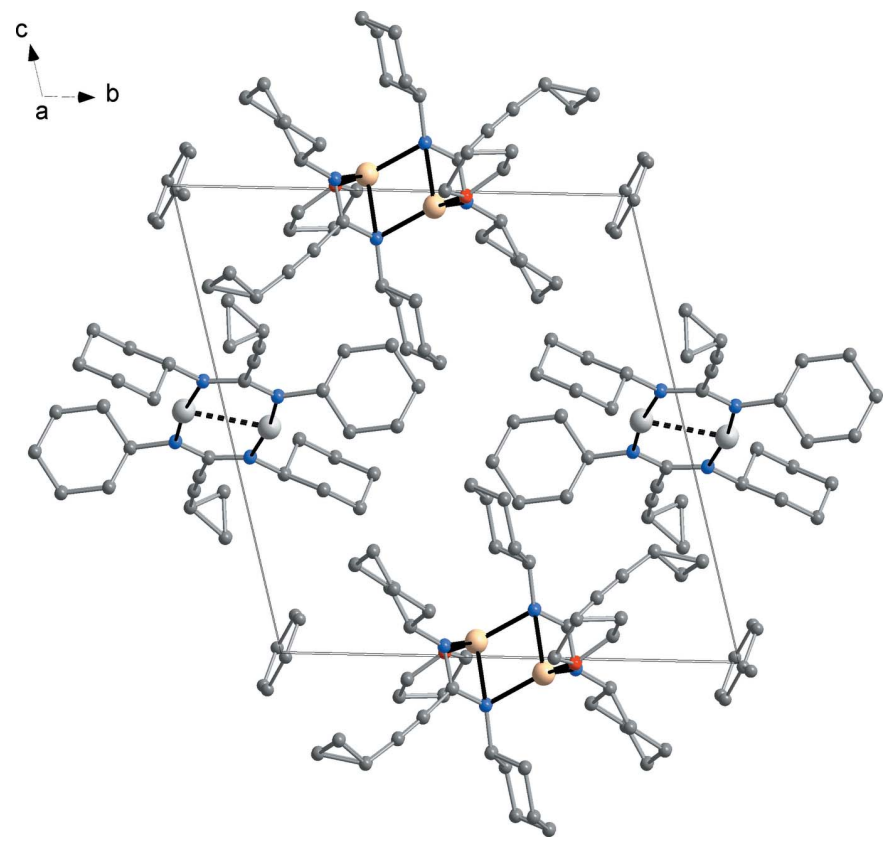

Figure 5

Crystal packing of silver(I) amidinate (2a), lithium amidinate (2b) and disordered toluene molecules in the co-crystal $\mathbf{2}$, viewed in a projection on (100).

components. Through this $\mu-\kappa N: \kappa N: \kappa N^{\prime}$-coordination mode of the amidinate ligands, a 'ladder' consisting of three fourmembered rings is formed. By coordination of a solvent THF molecule, a typical distorted tetrahedral coordination of the $\mathrm{Li}$ atom is completed. Just like in the case of the silver components $\mathbf{1}$ and $\mathbf{2 a}$, the $\mathrm{C}-\mathrm{N}$ bond lengths within the amidinate moiety are very similar with 1.321 (2) and 1.335 (2) A. The structural motif of ladder-type dimers is typical for this class of compounds and has frequently been observed for most of the previously characterized lithium amidinates and guanidinates (Stalke et al., 1992; Snaith \& Wright, 1995; Downard \& Chivers, 2001, Brown et al., 2008).

\section{Supramolecular features}

In both of the presented crystal structures, there are no specific intermolecular interactions. In compound 1 (Fig. 4), the closest intermolecular contacts exist between phenyl groups and isopropyl groups [min. $\mathrm{HC} \cdots \mathrm{CH}_{3} 3.79$ (1) $\AA$ ]. In the co-crystal 2 (Fig. 5), four silver amidinate molecules (2a) are situated on the centres of the four unit-cell edges perpendicular to (001) and four lithium amidinate molecules (2b) on the four edges perpendicular to (010). The four remaining unit-cell edges perpendicular to (100) are occupied by four disordered toluene molecules. The closest intermolecular contacts exist between the cyclopropyl moieties of

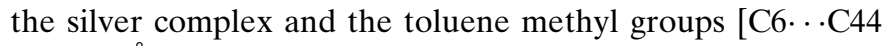
3.48 (1) $\AA$ ] , followed by cyclopropyl-cyclopropyl contacts between silver amidinate and lithium amidinate molecules [C5 …C24 3.57 (1) ̊]. 
Table 1

Experimental details.

1

Crystal data

Chemical formula

$M_{\text {r }}$

Crystal system, space group

Temperature $(\mathrm{K})$

$a, b, c(\AA)$

$\alpha, \beta, \gamma\left({ }^{\circ}\right)$

$V\left(\AA^{3}\right)$

Z

Radiation type

$\mu\left(\mathrm{mm}^{-1}\right)$

Crystal size (mm)

Data collection

Diffractometer

Absorption correction

$T_{\min }, T_{\max }$

No. of measured, independent and observed $[I>2 \sigma(I)]$ reflections

$R_{\text {int }}$

$(\sin \theta / \lambda)_{\max }\left(\AA^{-1}\right)$

Refinement

$R\left[F^{2}>2 \sigma\left(F^{2}\right)\right], w R\left(F^{2}\right), S$

No. of reflections

No. of parameters

No. of restraints

$\mathrm{H}$-atom treatment

$\Delta \rho_{\max }, \Delta \rho_{\min }\left(\mathrm{e} \AA^{-3}\right)$

\section{$\left[\mathrm{Ag}_{2}\left(\mathrm{C}_{13} \mathrm{H}_{19} \mathrm{~N}_{2}\right)_{2}\right]$}

622.34

Orthorhombic, $\mathrm{Pbca}$

153

11.7112 (6), 15.9238 (6), 14.8703 (6)

$90,90,90$

$2773.1(2)$

4

Mo $K \alpha$

1.43

$0.23 \times 0.21 \times 0.09$

Stoe IPDS 2T

Numerical ( $X$ - $A R E A$ and $X$-RED;

Stoe \& Cie, 2002)

$0.713,0.874$

$9641,3026,2360$

0.030

0.639

$0.024,0.047,0.99$

3026

150

0

$\mathrm{H}$-atom parameters constrained $0.34,-0.29$
$\left[\mathrm{Ag}_{2}\left(\mathrm{C}_{18} \mathrm{H}_{27} \mathrm{~N}_{2}\right)_{2}\right]\left[\mathrm{Li}_{2}\left(\mathrm{C}_{18} \mathrm{H}_{27} \mathrm{~N}_{2}\right)_{2}\left(\mathrm{C}_{4} \mathrm{H}_{8} \mathrm{O}\right)_{2}\right] \cdot-$

$\mathrm{C}_{7} \mathrm{H}_{8}$

1551.62

Triclinic, $P \overline{1}$

133

$10.5880(3), 14.5620(4), 14.9830$ (5)

99.871 (2), 102.825 (2), 106.538 (2)

2090.17 (11)

Mo $K \alpha$

0.52

$0.44 \times 0.29 \times 0.27$

Stoe IPDS 2T

$22444,9099,8214$

0.043

0.639

$0.028,0.073,1.03$

9099

461

12

$\mathrm{H}$-atom parameters constrained $0.40,-0.61$

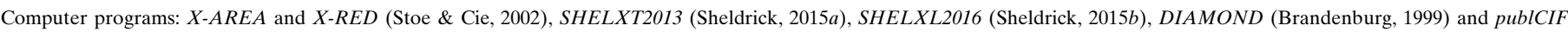
(Westrip, 2010).

\section{Synthesis and crystallization}

$\left[\mathrm{Ag}_{2}\left\{\mathrm{PhC}\left(\mathrm{N}^{i} \mathrm{Pr}\right)_{2}\right\}_{2}\right]$ (1) was obtained following a published procedure (Lim et al., 2003). Therefore, an in situ prepared solution of the lithium derivative $\operatorname{Li}\left\{\mathrm{PhC}\left(\mathrm{N}^{i} \mathrm{Pr}\right)_{2}\right\}$ (Sroor et al., 2013) in THF was treated with a stoichiometric amount of silver(I) chloride at room temperature (Fig. 6). Afterwards the solvent was removed in vacuo, the residue was extracted with toluene and the insoluble matter filtered off. After addition of an excess of $n$-pentane to the filtrate, large colorless crystals formed within few days at room temperature. ${ }^{1} \mathrm{H}$ NMR (400.1 MHz, THF- $\left.d_{8}, 298 \mathrm{~K}\right): \delta$ (p.p.m.) 7.45-7.04 $(3 \times m, 10 \mathrm{H}$, $\mathrm{CH} \mathrm{Ph}$ ), 3.21 (sept, $\left.4 \mathrm{H}, \mathrm{CH}{ }^{i} \mathrm{Pr}\right), 1.05$ (d, 24H, $\left.\mathrm{CH}_{3}{ }^{i} \mathrm{Pr}\right) .{ }^{13} \mathrm{C}$ NMR (100.6 MHz, THF- $\left.d_{8}, 298 \mathrm{~K}\right): \delta$ (p.p.m.) $170.4(\mathrm{NCN})$, 141.1 (ipso- $C \mathrm{Ph}$ ), 128.6 ( $\mathrm{CH} \mathrm{Ph}), 127.3$ ( $\mathrm{CH} \mathrm{Ph}), 126.7$ (para$\mathrm{CH} \mathrm{Ph}), 49.3\left(\mathrm{CH}^{i} \mathrm{Pr}\right), 28.1\left(\mathrm{CH}_{3}{ }^{i} \mathrm{Pr}\right)$.
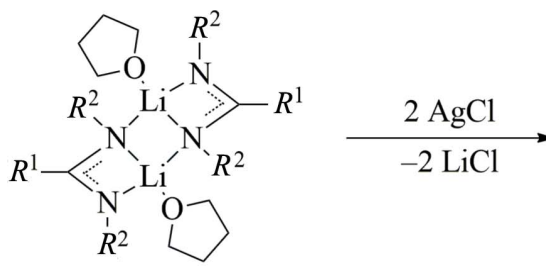

Figure 6

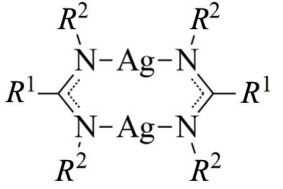

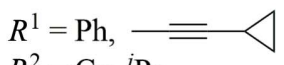

$R^{2}=\mathrm{Cy},{ }^{i} \mathrm{Pr}$

Synthesis of silver(I) amidinates from the related lithium derivatives.
Single crystals of the co-crystal (2) with composition $[\mathrm{Ag}\{c$ $\left.\left.\mathrm{C}_{3} \mathrm{H}_{5}-\mathrm{C} \equiv \mathrm{C}-\mathrm{C}(\mathrm{NCy})_{2}\right\}\right]_{2} \quad$ (2a) $\times \quad\left[\mathrm{Li}\left\{c-\mathrm{C}_{3} \mathrm{H}_{5}-\mathrm{C} \equiv \mathrm{C}-\right.\right.$ $\left.\left.\mathrm{C}(\mathrm{NCy})_{2}\right\}(\mathrm{THF})\right]_{2} \quad(\mathbf{2 b}) \times$ toluene were serendipitously obtained in an attempt to prepare the pure silver(I) derivative $\mathbf{2 a}$. The reaction of the in situ prepared lithium compound $\mathbf{2 b}$ (Sroor et al., 2013) with silver(I) chloride in THF analogous to the procedure described for compound $\mathbf{1}$ afforded a small quantity of colorless co-crystals of (2). Mp. = $393 \mathrm{~K} .{ }^{1} \mathrm{H}$ NMR (400.1 MHz, THF- $\left.d_{8}, 298 \mathrm{~K}\right): \delta$ (p.p.m.): 3.31-3.40 (m, 4H, CH Cy), 1.55-1.72 ( $\left.m, 20 \mathrm{H}, \mathrm{CH}_{2} \mathrm{Cy}\right), 1.34-1.40(m, 2 \mathrm{H}, \mathrm{CH}-$ $\left.\mathrm{C}_{3} \mathrm{H}_{5}\right), 1.09-1.23\left(\mathrm{~m}, 20 \mathrm{H}, \mathrm{CH}_{2} \mathrm{Cy}\right), 0.79-0.83\left(m, 4 \mathrm{H}, \mathrm{CH}_{2} c-\right.$ $\left.\mathrm{C}_{3} \mathrm{H}_{5}\right), 0.64-0.68\left(m, 4 \mathrm{H}, \mathrm{CH}_{2} c-\mathrm{C}_{3} \mathrm{H}_{5}\right) .{ }^{13} \mathrm{C} \mathrm{NMR}(100.6 \mathrm{MHz}$, $\left.\mathrm{THF}-d_{8}, 298 \mathrm{~K}\right): \delta$ (p.p.m.) $156.5(\mathrm{NCN}), 96.6(\mathrm{CH}-C \equiv \mathrm{C})$, $69.2(\mathrm{C} \equiv \mathrm{C}-\mathrm{C}), 58.8(\mathrm{CH} \mathrm{Cy}), 38.8\left(\mathrm{CH}_{2}, \mathrm{Cy}\right), 26.3\left(\mathrm{CH}_{2} \mathrm{Cy}\right)$, $8.83\left(\mathrm{CH}_{2} c-\mathrm{C}_{3} \mathrm{H}_{5}\right), 0.37\left(\mathrm{CH} c-\mathrm{C}_{3} \mathrm{H}_{5}\right)$.

\section{Refinement}

Crystal data, data collection and structure refinement details are summarized in Table 1 . All $\mathrm{H}$ atoms were fixed geometrically and refined using a riding model with $U_{\text {iso }}(\mathrm{H})=$ $1.2 U_{\text {eq }}(\mathrm{C}) . \mathrm{C}-\mathrm{H}$ distances in $\mathrm{CH}_{3}$ groups were constrained to $0.98 \AA$, those in $\mathrm{CH}_{2}$ groups to $0.99 \AA$ and those in $\mathrm{CH}$ groups to $1.00 \AA$. All $\mathrm{CH}_{3}$ groups were refined as freely rotating around the $\mathrm{C}-\mathrm{C}$ vector. 
For compound 2, the reflection (100) was partly obstructed by the beam stop and was therefore omitted from the refinement. The $U_{\mathrm{ij}}$ components of the C atoms of the THF molecule (C41-C44) were restrained to be similar for atoms closer than $1.7 \AA$ (SIMU restraint in SHELXL; the s.u. applied was $0.01 \AA^{2}$ ). The toluene molecule $(\mathrm{C} 41-\mathrm{C} 44)$ is located on an inversion center. Consequently, the methyl group (C44) and the para-H atom (H64) are disordered over two positions and were refined with a constrained site occupancy factor of 0.5 . The ipso-C and para-C atom (C42A and $\mathrm{C} 42 B$ ) were refined to be equal (EXYZ and EADP restraints in SHELXL).

\section{Acknowledgements}

This work was supported financially by the Otto-von-Guericke-Universität Magdeburg. SW holds a PhD studentship from the China Scholarship Council (CSC).

\section{References}

Aharonovich, S., Kapon, M., Botoshanski, M. \& Eisen, M. S. (2008). Organometallics, 27, 1869-1877.

Archibald, S. J., Alcock, N. W., Busch, D. H. \& Whitcomb, D. R. (2000). J. Cluster Sci. 11, 261-283.

Bailey, P. J. \& Pace, S. (2001). Coord. Chem. Rev. 214, 91-141.

Brandenburg, K. (1999). DIAMOND. University of Bonn, Germany.

Brown, D. J., Chisholm, M. H. \& Gallucci, J. C. (2008). Dalton Trans. pp. $1615-1624$.

Chlupatý, T., Padělková, A., Lyčka, A. \& Růžička, A. (2011). J. Organomet. Chem. 696, 2346-2354.

Collins, S. (2011). Coord. Chem. Rev. 255, 118-138.

Cure, J., Coppel, Y., Dammak, T., Fazzini, P. F., Mlayah, A., Chaudret, B. \& Fau, P. (2015). Langmuir, 31, 1362-1367.

Devi, A. (2013). Coord. Chem. Rev. 257, 3332-3384.

Downard, A. \& Chivers, T. (2001). Eur. J. Inorg. Chem. pp. $2193-$ 2201.

Edelmann, F. T. (2008). Adv. Organomet. Chem. 57, 183-352.

Edelmann, F. T. (2009). Chem. Soc. Rev. 38, 2253-2268.

Edelmann, F. T. (2012). Chem. Soc. Rev. 41, 7657-7672.

Edelmann, F. T. (2013). Adv. Organomet. Chem. 61, 55-374.
Gambarotta, S., Bracci, M., Floriani, C., Chiesi-Villa, A. \& Guastini, C. (1987). J. Chem. Soc. Dalton Trans. pp. 1883-1888.

Hitchcock, P. B., Lappert, M. F. \& Pierssens, L. J.-M. (1996). Chem. Commun. pp. 1189-1190.

Hong, J., Zhang, L., Wang, K., Chen, Z., Wu, L. \& Zhou, X. (2013). Organometallics, 32, 7312-7322.

Hope, H. \& Power, P. P. (1984). Inorg. Chem. 23, 936-937.

James, A. M., Laxman, R. K., Fronczek, F. R. \& Maverick, A. W. (1998). Inorg. Chem. 37, 3785-3791.

Jansen, M. (1987). Angew. Chem. Int. Ed. Engl. 26, 1098-1110.

Lane, A. C., Vollmer, M. V., Laber, C. H., Melgarejo, D. Y., Chiarella, G. M., Fackler, J. P. Jr, Yang, X., Baker, G. A. \& Walensky, J. R. (2014). Inorg. Chem. 53, 11357-11366.

Li, Z., Barry, S. T. \& Gordon, R. G. (2005). Inorg. Chem. 44, 17281735.

Li, X., Li, Y.-L., Chen, Y., Zou, Y., Zhuo, X.-B., Wu, Q.-Y., Zhao, Q.-J. \& Hu, H.-G. (2015). RSC Adv. 5, 94654-94657.

Liebing, P. \& Merzweiler, K. (2016). Z. Anorg. Allg. Chem. 642, 500507.

Lim, B. S., Rahtu, A., Park, J.-S. \& Gordon, R. G. (2003). Inorg. Chem. 42, 7951-7958.

Nevoralová, J., Chlupatý, T., Padělková, A. \& Růžička, A. (2013). J. Organomet. Chem. 745-746, 186-189.

Okano, A., James, R. C., Pierce, J. G., Xie, J. \& Boger, D. L. (2012). J. Am. Chem. Soc. 134, 8790-8793.

Pereshivko, O. P., Peshkov, V. A., Ermolat'ev, D. S., Van Hove, S., Van Hecke, K., Van Meervelt, L. \& Van der Eycken, E. V. (2011). Synthesis, pp. 1587-1594.

Sheldrick, G. M. (2015a). Acta Cryst. A71, 3-8.

Sheldrick, G. M. (2015b). Acta Cryst. C71, 3-8.

Snaith, R. \& Wright, D. S. (1995). In Lithium Chemistry, A Theoretical and Experimental Overview, edited by A. Sapse \& P. von R. Schleyer. New York: Wiley.

Sroor, F. M., Hrib, C. G., Hilfert, L. \& Edelmann, F. T. (2013). Z. Anorg. Allg. Chem. 639, 2390-2394.

Stalke, D., Wedler, M. \& Edelmann, F. T. (1992). J. Organomet. Chem. 431, C1-C5.

Stoe \& Cie (2002). $X$-AREA and $X$-RED. Stoe \& Cie, Darmstadt, Germany.

Trifonov, A. A. (2010). Coord. Chem. Rev. 254, 1327-1347.

Westrip, S. P. (2010). J. Appl. Cryst. 43, 920-925.

Whitehorne, T. J. J., Coyle, J. P., Mahmood, A., Monillas, W. H., Yap, G. P. A. \& Barry, S. T. (2011). Eur. J. Inorg. Chem. pp. 3240-3247. 


\section{supporting information}

Acta Cryst. (2016). E72, 1786-1790 [https://doi.org/10.1107/S2056989016017680]

\section{Crystal and molecular structures of two silver(I) amidinates, including an unexpected co-crystal with a lithium amidinate}

Sida Wang, Nicole Harmgarth, Phil Liebing and Frank T. Edelmann

Computing details

For both compounds, data collection: $X$-AREA (Stoe \& Cie, 2002); cell refinement: $X$-AREA (Stoe \& Cie, 2002); data reduction: $X$-AREA and $X$-RED (Stoe \& Cie, 2002); program(s) used to solve structure: SHELXT2013 (Sheldrick, 2015a); program(s) used to refine structure: SHELXL2016 (Sheldrick, 2015b); molecular graphics: DIAMOND (Brandenburg, 1999); software used to prepare material for publication: publCIF (Westrip, 2010).

(compound_1) Bis $\left[\mu-N^{1}, N^{2}-\right.$ bis(propan-2-yl)benzamidinato- $\left.\kappa^{2} N^{1}: N^{2}\right]$ disilver(I)

Crystal data

$\left[\mathrm{Ag}_{2}\left(\mathrm{C}_{13} \mathrm{H}_{19} \mathrm{~N}_{2}\right)_{2}\right]$

$M_{r}=622.34$

Orthorhombic, $\mathrm{Pbca}$

$a=11.7112(6) \AA$

$b=15.9238(6) \AA$

$c=14.8703(6) \AA$

$V=2773.1(2) \AA^{3}$

$Z=4$

$F(000)=1264$

Data collection

Stoe IPDS 2T

diffractometer

Radiation source: fine-focus sealed tube

Detector resolution: 6.67 pixels $\mathrm{mm}^{-1}$

area detector scans

Absorption correction: numerical

(X-AREA and X-RED; Stoe \& Cie, 2002)

$T_{\min }=0.713, T_{\max }=0.874$

Refinement

Refinement on $F^{2}$

Least-squares matrix: full

$R\left[F^{2}>2 \sigma\left(F^{2}\right)\right]=0.024$

$w R\left(F^{2}\right)=0.047$

$S=0.99$

3026 reflections

150 parameters

0 restraints

Primary atom site location: heavy-atom method
$D_{\mathrm{x}}=1.491 \mathrm{Mg} \mathrm{m}^{-3}$

Mo $K \alpha$ radiation, $\lambda=0.71073 \AA$

Cell parameters from 11797 reflections

$\theta=2.6-29.1^{\circ}$

$\mu=1.43 \mathrm{~mm}^{-1}$

$T=153 \mathrm{~K}$

Plate, colorless

$0.23 \times 0.21 \times 0.09 \mathrm{~mm}$

9641 measured reflections

3026 independent reflections

2360 reflections with $I>2 \sigma(I)$

$R_{\text {int }}=0.030$

$\theta_{\max }=27.0^{\circ}, \theta_{\min }=2.6^{\circ}$

$h=-12 \rightarrow 14$

$k=-17 \rightarrow 20$

$l=-18 \rightarrow 17$

Secondary atom site location: difference Fourier map

Hydrogen site location: inferred from neighbouring sites

$\mathrm{H}$-atom parameters constrained

$w=1 /\left[\sigma^{2}\left(F_{\mathrm{o}}^{2}\right)+(0.0238 P)^{2}\right]$

where $P=\left(F_{\mathrm{o}}^{2}+2 F_{\mathrm{c}}^{2}\right) / 3$

$(\Delta / \sigma)_{\max }=0.002$

$\Delta \rho_{\max }=0.34 \mathrm{e} \AA^{-3}$

$\Delta \rho_{\min }=-0.29 \mathrm{e} \AA^{-3}$ 
Extinction correction: SHELXL2016 (Sheldrick, 2015b),

$\mathrm{Fc}^{*}=\mathrm{kFc}\left[1+0.001 \mathrm{xFc}^{2} \lambda^{3} / \sin (2 \theta)\right]^{-1 / 4}$

Extinction coefficient: $0.00133(9)$

\section{Special details}

Geometry. All esds (except the esd in the dihedral angle between two 1.s. planes) are estimated using the full covariance matrix. The cell esds are taken into account individually in the estimation of esds in distances, angles and torsion angles; correlations between esds in cell parameters are only used when they are defined by crystal symmetry. An approximate (isotropic) treatment of cell esds is used for estimating esds involving l.s. planes.

Fractional atomic coordinates and isotropic or equivalent isotropic displacement parameters $\left(\AA^{2}\right)$

\begin{tabular}{|c|c|c|c|c|}
\hline & $x$ & $y$ & $z$ & $U_{\text {iso }} * / U_{\text {eq }}$ \\
\hline $\mathrm{C} 1$ & $0.89250(17)$ & 0.84957 (11) & $0.52126(14)$ & $0.0180(4)$ \\
\hline $\mathrm{C} 2$ & $0.84003(17)$ & $0.76359(12)$ & 0.53144 (14) & $0.0192(4)$ \\
\hline $\mathrm{C} 3$ & $0.72930(18)$ & $0.75332(14)$ & $0.56240(15)$ & $0.0269(5)$ \\
\hline $\mathrm{H} 1$ & 0.684814 & 0.801041 & 0.577986 & $0.032 *$ \\
\hline $\mathrm{C} 4$ & $0.6834(2)$ & $0.67346(15)$ & $0.57063(17)$ & 0.0339 (6) \\
\hline $\mathrm{H} 2$ & 0.607343 & 0.666906 & 0.591646 & $0.041^{*}$ \\
\hline $\mathrm{C} 5$ & $0.7464(2)$ & $0.60378(15)$ & $0.54883(17)$ & $0.0347(6)$ \\
\hline H3 & 0.714312 & 0.549257 & 0.554655 & $0.042 *$ \\
\hline C6 & $0.8568(2)$ & $0.61370(14)$ & $0.51837(17)$ & $0.0347(6)$ \\
\hline $\mathrm{H} 4$ & 0.901066 & 0.565694 & 0.503496 & $0.042 *$ \\
\hline $\mathrm{C} 7$ & $0.9036(2)$ & $0.69336(13)$ & 0.50927 (16) & $0.0279(5)$ \\
\hline H5 & 0.979524 & 0.699645 & 0.487771 & $0.034^{*}$ \\
\hline $\mathrm{C} 8$ & $0.9646(2)$ & 0.83508 (14) & $0.67386(15)$ & $0.0307(5)$ \\
\hline H6 & 0.926464 & 0.779127 & 0.668444 & $0.037^{*}$ \\
\hline C9 & $1.0893(3)$ & $0.82211(19)$ & $0.6933(2)$ & $0.0519(8)$ \\
\hline H7 & 1.097759 & 0.790334 & 0.749337 & $0.062 *$ \\
\hline H9 & 1.126925 & 0.876767 & 0.699461 & $0.062 *$ \\
\hline H8 & 1.124389 & 0.790784 & 0.643782 & $0.062 *$ \\
\hline $\mathrm{C} 10$ & $0.9090(3)$ & $0.8841(2)$ & 0.7489 (2) & $0.0657(10)$ \\
\hline H12 & 0.915326 & 0.852442 & 0.805200 & $0.079^{*}$ \\
\hline H10 & 0.828196 & 0.893125 & 0.734664 & $0.079^{*}$ \\
\hline H11 & 0.947262 & 0.938437 & 0.755509 & $0.079 *$ \\
\hline C11 & $0.8084(2)$ & $0.85689(12)$ & $0.37151(15)$ & $0.0255(5)$ \\
\hline H13 & 0.793586 & 0.795930 & 0.382647 & $0.031^{*}$ \\
\hline $\mathrm{C} 12$ & $0.6952(2)$ & 0.90348 (17) & $0.3702(2)$ & $0.0431(7)$ \\
\hline H15 & 0.648800 & 0.883280 & 0.319816 & $0.052 *$ \\
\hline H16 & 0.709247 & 0.963797 & 0.363193 & $0.052 *$ \\
\hline H14 & 0.654535 & 0.893414 & 0.426792 & $0.052 *$ \\
\hline $\mathrm{C} 13$ & 0.8693 (3) & $0.86641(16)$ & $0.28221(16)$ & 0.0398 (6) \\
\hline H19 & 0.821450 & 0.843287 & 0.234130 & $0.048^{*}$ \\
\hline H18 & 0.942018 & 0.836057 & 0.284187 & $0.048^{*}$ \\
\hline H17 & 0.883690 & 0.926043 & 0.270543 & $0.048 *$ \\
\hline N1 & $0.95209(16)$ & $0.88054(10)$ & $0.58907(12)$ & $0.0225(4)$ \\
\hline $\mathrm{N} 2$ & $0.88089(15)$ & $0.88965(10)$ & $0.44393(12)$ & 0.0194 (4) \\
\hline $\mathrm{AG}$ & $1.04222(2)$ & $0.99411(2)$ & $0.58280(2)$ & $0.01974(6)$ \\
\hline
\end{tabular}


Atomic displacement parameters $\left(\AA^{2}\right)$

\begin{tabular}{lllllll}
\hline & $U^{11}$ & $U^{22}$ & $U^{33}$ & $U^{12}$ & $U^{13}$ & $U^{23}$ \\
\hline C1 & $0.0154(10)$ & $0.0156(8)$ & $0.0229(10)$ & $-0.0005(7)$ & $0.0023(8)$ & $-0.0010(7)$ \\
C2 & $0.0205(11)$ & $0.0201(9)$ & $0.0172(10)$ & $-0.0048(8)$ & $-0.0028(8)$ & $0.0025(8)$ \\
C3 & $0.0226(13)$ & $0.0312(10)$ & $0.0269(11)$ & $-0.0027(9)$ & $-0.0015(9)$ & $0.0032(9)$ \\
C4 & $0.0260(12)$ & $0.0422(12)$ & $0.0334(14)$ & $-0.0165(10)$ & $-0.0069(10)$ & $0.0108(11)$ \\
C5 & $0.0462(15)$ & $0.0267(10)$ & $0.0312(13)$ & $-0.0194(10)$ & $-0.0126(12)$ & $0.0066(9)$ \\
C6 & $0.0454(15)$ & $0.0207(10)$ & $0.0381(14)$ & $-0.0038(10)$ & $-0.0069(12)$ & $-0.0004(10)$ \\
C7 & $0.0317(13)$ & $0.0213(9)$ & $0.0308(13)$ & $-0.0027(9)$ & $0.0007(10)$ & $0.0003(9)$ \\
C8 & $0.0435(15)$ & $0.0276(10)$ & $0.0209(11)$ & $-0.0129(11)$ & $-0.0057(11)$ & $0.0074(9)$ \\
C9 & $0.057(2)$ & $0.0559(17)$ & $0.0424(17)$ & $0.0170(14)$ & $-0.0130(15)$ & $0.0194(14)$ \\
C10 & $0.068(2)$ & $0.101(3)$ & $0.0281(15)$ & $0.0053(19)$ & $0.0150(17)$ & $0.0124(19)$ \\
C11 & $0.0309(12)$ & $0.0185(9)$ & $0.0270(12)$ & $-0.0041(9)$ & $-0.0118(10)$ & $-0.0007(8)$ \\
C12 & $0.0332(14)$ & $0.0476(14)$ & $0.0484(17)$ & $0.0024(12)$ & $-0.0179(13)$ & $-0.0024(13)$ \\
C13 & $0.0567(18)$ & $0.0383(12)$ & $0.0243(12)$ & $-0.0063(13)$ & $-0.0097(12)$ & $-0.0104(10)$ \\
N1 & $0.0273(9)$ & $0.0213(7)$ & $0.0190(9)$ & $-0.0074(7)$ & $-0.0039(8)$ & $0.0036(7)$ \\
N2 & $0.0213(9)$ & $0.0169(7)$ & $0.0200(9)$ & $-0.0028(7)$ & $-0.0058(7)$ & $0.0006(6)$ \\
AG & $0.02505(8)$ & $0.01632(8)$ & $0.01784(8)$ & $-0.00466(6)$ & $-0.00495(6)$ & $0.00110(6)$ \\
& & & & & &
\end{tabular}

Geometric parameters $\left(\AA,{ }^{\circ}\right)$

\begin{tabular}{|c|c|c|c|}
\hline $\mathrm{C} 1-\mathrm{N} 1$ & $1.322(3)$ & $\mathrm{C} 9-\mathrm{H} 9$ & 0.9800 \\
\hline $\mathrm{C} 1-\mathrm{N} 2$ & $1.322(3)$ & $\mathrm{C} 9-\mathrm{H} 8$ & 0.9800 \\
\hline $\mathrm{C} 1-\mathrm{C} 2$ & $1.508(3)$ & $\mathrm{C} 10-\mathrm{H} 12$ & 0.9800 \\
\hline $\mathrm{C} 2-\mathrm{C} 7$ & $1.383(3)$ & $\mathrm{C} 10-\mathrm{H} 10$ & 0.9800 \\
\hline $\mathrm{C} 2-\mathrm{C} 3$ & $1.386(3)$ & $\mathrm{C} 10-\mathrm{H} 11$ & 0.9800 \\
\hline $\mathrm{C} 3-\mathrm{C} 4$ & $1.386(3)$ & $\mathrm{C} 11-\mathrm{N} 2$ & $1.467(3)$ \\
\hline $\mathrm{C} 3-\mathrm{H} 1$ & 0.9500 & $\mathrm{C} 11-\mathrm{C} 13$ & $1.514(4)$ \\
\hline $\mathrm{C} 4-\mathrm{C} 5$ & $1.371(4)$ & $\mathrm{C} 11-\mathrm{C} 12$ & $1.520(3)$ \\
\hline $\mathrm{C} 4-\mathrm{H} 2$ & 0.9500 & $\mathrm{C} 11-\mathrm{H} 13$ & 1.0000 \\
\hline $\mathrm{C} 5-\mathrm{C} 6$ & $1.379(4)$ & $\mathrm{C} 12-\mathrm{H} 15$ & 0.9800 \\
\hline $\mathrm{C} 5-\mathrm{H} 3$ & 0.9500 & $\mathrm{C} 12-\mathrm{H} 16$ & 0.9800 \\
\hline $\mathrm{C} 6-\mathrm{C} 7$ & $1.389(3)$ & $\mathrm{C} 12-\mathrm{H} 14$ & 0.9800 \\
\hline $\mathrm{C} 6-\mathrm{H} 4$ & 0.9500 & C13-H19 & 0.9800 \\
\hline $\mathrm{C} 7-\mathrm{H} 5$ & 0.9500 & C13-H18 & 0.9800 \\
\hline $\mathrm{C} 8-\mathrm{N} 1$ & $1.461(3)$ & $\mathrm{C} 13-\mathrm{H} 17$ & 0.9800 \\
\hline $\mathrm{C} 8-\mathrm{C} 9$ & $1.502(4)$ & $\mathrm{N} 1-\mathrm{AG}$ & $2.0959(16)$ \\
\hline $\mathrm{C} 8-\mathrm{C} 10$ & $1.509(4)$ & $\mathrm{N} 2-\mathrm{AG}^{\mathrm{i}}$ & $2.0965(16)$ \\
\hline $\mathrm{C} 8-\mathrm{H} 6$ & 1.0000 & $\mathrm{AG}-\mathrm{N} 2^{\mathrm{i}}$ & $2.0965(16)$ \\
\hline $\mathrm{C} 9-\mathrm{H} 7$ & 0.9800 & $\mathrm{AG}-\mathrm{AG}^{\mathrm{i}}$ & $2.6604(3)$ \\
\hline $\mathrm{N} 1-\mathrm{C} 1-\mathrm{N} 2$ & $122.53(17)$ & $\mathrm{C} 8-\mathrm{C} 10-\mathrm{H} 12$ & 109.5 \\
\hline $\mathrm{N} 1-\mathrm{C} 1-\mathrm{C} 2$ & $118.50(18)$ & $\mathrm{C} 8-\mathrm{C} 10-\mathrm{H} 10$ & 109.5 \\
\hline $\mathrm{N} 2-\mathrm{C} 1-\mathrm{C} 2$ & 118.92 (18) & $\mathrm{H} 12-\mathrm{C} 10-\mathrm{H} 10$ & 109.5 \\
\hline $\mathrm{C} 7-\mathrm{C} 2-\mathrm{C} 3$ & $119.2(2)$ & $\mathrm{C} 8-\mathrm{C} 10-\mathrm{H} 11$ & 109.5 \\
\hline $\mathrm{C} 7-\mathrm{C} 2-\mathrm{C} 1$ & 119.38 (19) & $\mathrm{H} 12-\mathrm{C} 10-\mathrm{H} 11$ & 109.5 \\
\hline $\mathrm{C} 3-\mathrm{C} 2-\mathrm{C} 1$ & 121.45 (19) & $\mathrm{H} 10-\mathrm{C} 10-\mathrm{H} 11$ & 109.5 \\
\hline
\end{tabular}




\begin{tabular}{|c|c|c|c|}
\hline $\mathrm{C} 2-\mathrm{C} 3-\mathrm{C} 4$ & $120.0(2)$ & $\mathrm{N} 2-\mathrm{C} 11-\mathrm{C} 13$ & $109.64(19)$ \\
\hline $\mathrm{C} 2-\mathrm{C} 3-\mathrm{H} 1$ & 120.0 & $\mathrm{~N} 2-\mathrm{C} 11-\mathrm{C} 12$ & $109.90(19)$ \\
\hline $\mathrm{C} 4-\mathrm{C} 3-\mathrm{H} 1$ & 120.0 & $\mathrm{C} 13-\mathrm{C} 11-\mathrm{C} 12$ & $110.5(2)$ \\
\hline $\mathrm{C} 5-\mathrm{C} 4-\mathrm{C} 3$ & $120.9(2)$ & $\mathrm{N} 2-\mathrm{C} 11-\mathrm{H} 13$ & 108.9 \\
\hline $\mathrm{C} 5-\mathrm{C} 4-\mathrm{H} 2$ & 119.6 & $\mathrm{C} 13-\mathrm{C} 11-\mathrm{H} 13$ & 108.9 \\
\hline $\mathrm{C} 3-\mathrm{C} 4-\mathrm{H} 2$ & 119.6 & $\mathrm{C} 12-\mathrm{C} 11-\mathrm{H} 13$ & 108.9 \\
\hline $\mathrm{C} 4-\mathrm{C} 5-\mathrm{C} 6$ & $119.3(2)$ & $\mathrm{C} 11-\mathrm{C} 12-\mathrm{H} 15$ & 109.5 \\
\hline $\mathrm{C} 4-\mathrm{C} 5-\mathrm{H} 3$ & 120.4 & $\mathrm{C} 11-\mathrm{C} 12-\mathrm{H} 16$ & 109.5 \\
\hline $\mathrm{C} 6-\mathrm{C} 5-\mathrm{H} 3$ & 120.4 & $\mathrm{H} 15-\mathrm{C} 12-\mathrm{H} 16$ & 109.5 \\
\hline $\mathrm{C} 5-\mathrm{C} 6-\mathrm{C} 7$ & $120.5(2)$ & $\mathrm{C} 11-\mathrm{C} 12-\mathrm{H} 14$ & 109.5 \\
\hline $\mathrm{C} 5-\mathrm{C} 6-\mathrm{H} 4$ & 119.8 & $\mathrm{H} 15-\mathrm{C} 12-\mathrm{H} 14$ & 109.5 \\
\hline $\mathrm{C} 7-\mathrm{C} 6-\mathrm{H} 4$ & 119.8 & $\mathrm{H} 16-\mathrm{C} 12-\mathrm{H} 14$ & 109.5 \\
\hline $\mathrm{C} 2-\mathrm{C} 7-\mathrm{C} 6$ & $120.2(2)$ & $\mathrm{C} 11-\mathrm{C} 13-\mathrm{H} 19$ & 109.5 \\
\hline $\mathrm{C} 2-\mathrm{C} 7-\mathrm{H} 5$ & 119.9 & $\mathrm{C} 11-\mathrm{C} 13-\mathrm{H} 18$ & 109.5 \\
\hline $\mathrm{C} 6-\mathrm{C} 7-\mathrm{H} 5$ & 119.9 & $\mathrm{H} 19-\mathrm{C} 13-\mathrm{H} 18$ & 109.5 \\
\hline $\mathrm{N} 1-\mathrm{C} 8-\mathrm{C} 9$ & $109.4(2)$ & $\mathrm{C} 11-\mathrm{C} 13-\mathrm{H} 17$ & 109.5 \\
\hline $\mathrm{N} 1-\mathrm{C} 8-\mathrm{C} 10$ & $109.8(2)$ & $\mathrm{H} 19-\mathrm{C} 13-\mathrm{H} 17$ & 109.5 \\
\hline $\mathrm{C} 9-\mathrm{C} 8-\mathrm{C} 10$ & $110.4(2)$ & $\mathrm{H} 18-\mathrm{C} 13-\mathrm{H} 17$ & 109.5 \\
\hline $\mathrm{N} 1-\mathrm{C} 8-\mathrm{H} 6$ & 109.1 & $\mathrm{C} 1-\mathrm{N} 1-\mathrm{C} 8$ & $121.76(16)$ \\
\hline $\mathrm{C} 9-\mathrm{C} 8-\mathrm{H} 6$ & 109.1 & $\mathrm{C} 1-\mathrm{N} 1-\mathrm{AG}$ & $123.63(13)$ \\
\hline $\mathrm{C} 10-\mathrm{C} 8-\mathrm{H} 6$ & 109.1 & $\mathrm{C} 8-\mathrm{N} 1-\mathrm{AG}$ & $114.54(13)$ \\
\hline $\mathrm{C} 8-\mathrm{C} 9-\mathrm{H} 7$ & 109.5 & $\mathrm{C} 1-\mathrm{N} 2-\mathrm{C} 11$ & $121.76(17)$ \\
\hline $\mathrm{C} 8-\mathrm{C} 9-\mathrm{H} 9$ & 109.5 & $\mathrm{C} 1-\mathrm{N} 2-\mathrm{AG}^{\mathrm{i}}$ & $123.15(13)$ \\
\hline $\mathrm{H} 7-\mathrm{C} 9-\mathrm{H} 9$ & 109.5 & $\mathrm{C} 11-\mathrm{N} 2-\mathrm{AG}^{\mathrm{i}}$ & $115.04(12)$ \\
\hline $\mathrm{C} 8-\mathrm{C} 9-\mathrm{H} 8$ & 109.5 & $\mathrm{~N} 1-\mathrm{AG}-\mathrm{N} 2^{\mathrm{i}}$ & $170.58(7)$ \\
\hline $\mathrm{H} 7-\mathrm{C} 9-\mathrm{H} 8$ & 109.5 & $\mathrm{~N} 1-\mathrm{AG}-\mathrm{AG}^{\mathrm{i}}$ & $85.12(5)$ \\
\hline $\mathrm{H} 9-\mathrm{C} 9-\mathrm{H} 8$ & 109.5 & $\mathrm{~N} 2 \mathrm{i}-\mathrm{AG}-\mathrm{AG}^{\mathrm{i}}$ & $85.52(5)$ \\
\hline $\mathrm{N} 1-\mathrm{C} 1-\mathrm{C} 2-\mathrm{C} 7$ & $-87.4(3)$ & $\mathrm{N} 2-\mathrm{C} 1-\mathrm{N} 1-\mathrm{AG}$ & $-2.7(3)$ \\
\hline $\mathrm{N} 2-\mathrm{C} 1-\mathrm{C} 2-\mathrm{C} 7$ & $90.1(3)$ & $\mathrm{C} 2-\mathrm{C} 1-\mathrm{N} 1-\mathrm{AG}$ & $174.65(14)$ \\
\hline $\mathrm{N} 1-\mathrm{C} 1-\mathrm{C} 2-\mathrm{C} 3$ & $92.7(3)$ & $\mathrm{C} 9-\mathrm{C} 8-\mathrm{N} 1-\mathrm{C} 1$ & $122.8(2)$ \\
\hline $\mathrm{N} 2-\mathrm{C} 1-\mathrm{C} 2-\mathrm{C} 3$ & $-89.8(3)$ & $\mathrm{C} 10-\mathrm{C} 8-\mathrm{N} 1-\mathrm{C} 1$ & $-116.0(3)$ \\
\hline $\mathrm{C} 7-\mathrm{C} 2-\mathrm{C} 3-\mathrm{C} 4$ & $-0.2(3)$ & $\mathrm{C} 9-\mathrm{C} 8-\mathrm{N} 1-\mathrm{AG}$ & $-54.4(2)$ \\
\hline $\mathrm{C} 1-\mathrm{C} 2-\mathrm{C} 3-\mathrm{C} 4$ & $179.7(2)$ & $\mathrm{C} 10-\mathrm{C} 8-\mathrm{N} 1-\mathrm{AG}$ & $66.9(3)$ \\
\hline $\mathrm{C} 2-\mathrm{C} 3-\mathrm{C} 4-\mathrm{C} 5$ & $0.3(4)$ & $\mathrm{N} 1-\mathrm{C} 1-\mathrm{N} 2-\mathrm{C} 11$ & $-176.00(19)$ \\
\hline $\mathrm{C} 3-\mathrm{C} 4-\mathrm{C} 5-\mathrm{C} 6$ & $0.0(4)$ & $\mathrm{C} 2-\mathrm{C} 1-\mathrm{N} 2-\mathrm{C} 11$ & $6.6(3)$ \\
\hline $\mathrm{C} 4-\mathrm{C} 5-\mathrm{C} 6-\mathrm{C} 7$ & $-0.4(4)$ & $\mathrm{N} 1-\mathrm{C} 1-\mathrm{N} 2-\mathrm{AG}^{\mathrm{i}}$ & $1.4(3)$ \\
\hline $\mathrm{C} 3-\mathrm{C} 2-\mathrm{C} 7-\mathrm{C} 6$ & $-0.2(3)$ & $\mathrm{C} 2-\mathrm{C} 1-\mathrm{N} 2-\mathrm{AG}^{\mathrm{i}}$ & $-175.99(14)$ \\
\hline $\mathrm{C} 1-\mathrm{C} 2-\mathrm{C} 7-\mathrm{C} 6$ & $179.9(2)$ & $\mathrm{C} 13-\mathrm{C} 11-\mathrm{N} 2-\mathrm{C} 1$ & $-137.0(2)$ \\
\hline $\mathrm{C} 5-\mathrm{C} 6-\mathrm{C} 7-\mathrm{C} 2$ & $0.5(4)$ & $\mathrm{C} 12-\mathrm{C} 11-\mathrm{N} 2-\mathrm{C} 1$ & $101.3(2)$ \\
\hline $\mathrm{N} 2-\mathrm{C} 1-\mathrm{N} 1-\mathrm{C} 8$ & $-179.6(2)$ & $\mathrm{C} 13-\mathrm{C} 11-\mathrm{N} 2-\mathrm{AG}^{\mathrm{i}}$ & $45.4(2)$ \\
\hline $\mathrm{C} 2-\mathrm{C} 1-\mathrm{N} 1-\mathrm{C} 8$ & $-2.2(3)$ & $\mathrm{C} 12-\mathrm{C} 11-\mathrm{N} 2-\mathrm{AG}^{\mathrm{i}}$ & $-76.2(2)$ \\
\hline
\end{tabular}

Symmetry code: (i) $-x+2,-y+2,-z+1$. 
(compound_2) Bis $\left(\mu-N^{1}, N^{2}\right.$-dicyclohexyl-3-cyclopropylpropynamidinato- $\left.\kappa^{2} N^{1}: N^{2}\right) \operatorname{disilver}(\mathrm{I}) \operatorname{bis}\left(\mu-N^{1}, N^{2}\right.$ dicyclohexyl-3-cyclopropylpropynamidinato- $\kappa^{3} N^{1}, N^{2}: N^{1}$ )bis(tetrahydrofuran- $\kappa O$ ) lithium(I) toluene monosolvate

Crystal data

$\begin{array}{ll}{\left[\mathrm{Ag}_{2}\left(\mathrm{C}_{18} \mathrm{H}_{27} \mathrm{~N}_{2}\right)_{2}\right]\left[\mathrm{Li}_{2}\left(\mathrm{C}_{18} \mathrm{H}_{27} \mathrm{~N}_{2}\right)_{2}\left(\mathrm{C}_{4} \mathrm{H}_{8} \mathrm{O}\right)_{2}\right] \cdot \mathrm{C}_{7} \mathrm{H}_{8}} & Z=1 \\ M_{r}=1551.62 & F(000)=826 \\ \text { Triclinic, } P \overline{1} & D_{\mathrm{x}}=1.233 \mathrm{Mg} \mathrm{m}^{-3} \\ a=10.5880(3) \AA & \text { Mo } K \alpha \text { radiation, } \lambda=0.71073 \AA \\ b=14.5620(4) \AA & \text { Cell parameters from } 27393 \text { reflections } \\ c=14.9830(5) \AA & \theta=2.1-29.2^{\circ} \\ \alpha=99.871(2)^{\circ} & \mu=0.52 \mathrm{~mm}^{-1} \\ \beta=102.825(2)^{\circ} & T=133 \mathrm{~K} \\ \gamma=106.538(2)^{\circ} & \text { Rod, colorless } \\ V=2090.17(11) \AA^{3} & 0.44 \times 0.29 \times 0.27 \mathrm{~mm}\end{array}$

\section{Data collection}

Stoe IPDS 2T diffractometer

Radiation source: fine-focus sealed tube Detector resolution: 6.67 pixels $\mathrm{mm}^{-1}$ area detector scans 22444 measured reflections 9099 independent reflections

8214 reflections with $I>2 \sigma(I)$

$R_{\text {int }}=0.043$

$\theta_{\max }=27.0^{\circ}, \theta_{\min }=2.1^{\circ}$

$h=-12 \rightarrow 13$

$k=-18 \rightarrow 18$

$l=-19 \rightarrow 19$

\section{Refinement}

Refinement on $F^{2}$

Least-squares matrix: full

$R\left[F^{2}>2 \sigma\left(F^{2}\right)\right]=0.028$

$w R\left(F^{2}\right)=0.073$

$S=1.03$

9099 reflections

461 parameters

12 restraints

Primary atom site location: heavy-atom method
Secondary atom site location: difference Fourier map

Hydrogen site location: inferred from neighbouring sites

$\mathrm{H}$-atom parameters constrained

$w=1 /\left[\sigma^{2}\left(F_{\mathrm{o}}^{2}\right)+(0.0398 P)^{2}+0.4823 P\right]$

where $P=\left(F_{\mathrm{o}}^{2}+2 F_{\mathrm{c}}{ }^{2}\right) / 3$

$(\Delta / \sigma)_{\max }=0.003$

$\Delta \rho_{\max }=0.40 \mathrm{e} \AA^{-3}$

$\Delta \rho_{\min }=-0.61 \mathrm{e} \AA^{-3}$

Special details

Geometry. All esds (except the esd in the dihedral angle between two 1.s. planes) are estimated using the full covariance matrix. The cell esds are taken into account individually in the estimation of esds in distances, angles and torsion angles; correlations between esds in cell parameters are only used when they are defined by crystal symmetry. An approximate (isotropic) treatment of cell esds is used for estimating esds involving l.s. planes.

Fractional atomic coordinates and isotropic or equivalent isotropic displacement parameters $\left(\AA^{2}\right)$

\begin{tabular}{llllll}
\hline & $x$ & $y$ & $z$ & $U_{\text {iso }}^{*} / U_{\text {eq }}$ & Occ. $(<1)$ \\
\hline C1 & $0.72575(17)$ & $0.93982(12)$ & $0.41483(12)$ & $0.0218(3)$ & \\
C2 & $0.57831(19)$ & $0.90959(12)$ & $0.37327(13)$ & $0.0246(3)$ & \\
C3 & $0.45722(19)$ & $0.89050(13)$ & $0.34225(14)$ & $0.0288(4)$ & \\
C4 & $0.3132(2)$ & $0.87385(16)$ & $0.30639(16)$ & $0.0372(4)$ & \\
H1 & 0.250264 & 0.804282 & 0.293635 & $0.045^{*}$ & \\
C5 & $0.2590(3)$ & $0.9540(2)$ & $0.33985(19)$ & $0.0530(6)$ & \\
H3 & 0.166275 & 0.933659 & 0.349156 & $0.064^{*}$ &
\end{tabular}




\begin{tabular}{|c|c|c|c|c|}
\hline $\mathrm{H} 2$ & 0.325410 & 1.015476 & 0.385331 & $0.064 *$ \\
\hline C6 & 0.2683 & $0.9347(2)$ & $0.24215(18)$ & $0.0517(6)$ \\
\hline H4 & 0.340640 & 0.984016 & 0.226610 & $0.062^{*}$ \\
\hline H5 & 0.181473 & 0.902183 & 0.190427 & $0.062 *$ \\
\hline $\mathrm{C} 7$ & $0.68836(18)$ & $0.77293(12)$ & $0.43251(13)$ & $0.0250(3)$ \\
\hline H6 & 0.591076 & 0.771776 & 0.420229 & $0.030^{*}$ \\
\hline $\mathrm{C} 8$ & $0.6996(2)$ & $0.70587(14)$ & $0.34709(15)$ & $0.0364(5)$ \\
\hline H8 & 0.796825 & 0.710104 & 0.356146 & $0.044 *$ \\
\hline H7 & 0.670205 & 0.728808 & 0.289972 & $0.044^{*}$ \\
\hline C9 & $0.6106(3)$ & $0.59800(16)$ & $0.3322(2)$ & $0.0523(7)$ \\
\hline H9 & 0.512424 & 0.592466 & 0.315540 & $0.063 *$ \\
\hline H10 & 0.625175 & 0.555915 & 0.278802 & $0.063^{*}$ \\
\hline $\mathrm{C} 10$ & $0.6448(3)$ & $0.56124(16)$ & $0.4195(2)$ & $0.0543(7)$ \\
\hline H12 & 0.739052 & 0.558114 & 0.431136 & $0.065^{*}$ \\
\hline H11 & 0.580377 & 0.493464 & 0.409075 & $0.065^{*}$ \\
\hline C11 & $0.6355(2)$ & $0.62794(16)$ & $0.50555(18)$ & $0.0436(5)$ \\
\hline H13 & 0.665684 & 0.604587 & 0.562243 & $0.052 *$ \\
\hline H14 & 0.538532 & 0.624002 & 0.497493 & $0.052 *$ \\
\hline $\mathrm{C} 12$ & $0.7243(2)$ & $0.73533(15)$ & $0.52052(16)$ & $0.0361(4)$ \\
\hline H16 & 0.710784 & 0.777303 & 0.574501 & $0.043^{*}$ \\
\hline H15 & 0.822439 & 0.740615 & 0.536326 & $0.043 *$ \\
\hline $\mathrm{C} 13$ & $0.73199(18)$ & $1.09818(12)$ & $0.38343(12)$ & $0.0228(3)$ \\
\hline H17 & 0.651330 & 1.058023 & 0.327716 & $0.027^{*}$ \\
\hline C14 & $0.6799(2)$ & $1.14824(14)$ & $0.45820(14)$ & $0.0304(4)$ \\
\hline H18 & 0.758111 & 1.186566 & 0.514822 & $0.037^{*}$ \\
\hline H19 & 0.614428 & 1.097199 & 0.477180 & $0.037^{*}$ \\
\hline $\mathrm{C} 15$ & $0.6084(2)$ & $1.21748(16)$ & $0.42102(17)$ & $0.0398(5)$ \\
\hline $\mathrm{H} 21$ & 0.525174 & 1.178218 & 0.368054 & $0.048^{*}$ \\
\hline $\mathrm{H} 20$ & 0.579014 & 1.251029 & 0.471881 & $0.048^{*}$ \\
\hline $\mathrm{C} 16$ & $0.7029(2)$ & $1.29438(15)$ & $0.38732(16)$ & $0.0400(5)$ \\
\hline $\mathrm{H} 22$ & 0.780227 & 1.339007 & 0.441950 & $0.048^{*}$ \\
\hline $\mathrm{H} 23$ & 0.651693 & 1.334757 & 0.359347 & $0.048 *$ \\
\hline $\mathrm{C} 17$ & $0.7593(2)$ & $1.24632(14)$ & $0.31411(15)$ & $0.0350(4)$ \\
\hline $\mathrm{H} 25$ & 0.826715 & 1.298382 & 0.297605 & $0.042 *$ \\
\hline H24 & 0.683240 & 1.209012 & 0.255944 & $0.042 *$ \\
\hline $\mathrm{C} 18$ & $0.8288(2)$ & $1.17612(13)$ & $0.35133(14)$ & 0.0293 (4) \\
\hline $\mathrm{H} 27$ & 0.859313 & 1.143032 & 0.300877 & $0.035^{*}$ \\
\hline $\mathrm{H} 26$ & 0.911196 & 1.214723 & 0.405123 & $0.035^{*}$ \\
\hline C19 & $0.94671(19)$ & $0.64290(12)$ & $0.06927(13)$ & $0.0254(4)$ \\
\hline $\mathrm{C} 20$ & $0.92323(19)$ & $0.72135(13)$ & $0.13212(13)$ & $0.0261(4)$ \\
\hline $\mathrm{C} 21$ & 0.90549 (19) & $0.78792(13)$ & $0.17990(13)$ & $0.0255(4)$ \\
\hline $\mathrm{C} 22$ & $0.88536(19)$ & $0.87128(13)$ & $0.23412(13)$ & $0.0259(4)$ \\
\hline $\mathrm{H} 28$ & 0.887959 & 0.871143 & 0.301222 & $0.031 *$ \\
\hline $\mathrm{C} 23$ & $0.7900(2)$ & $0.91816(15)$ & $0.18509(15)$ & $0.0342(4)$ \\
\hline $\mathrm{H} 30$ & 0.744110 & 0.889465 & 0.116586 & $0.041 *$ \\
\hline $\mathrm{H} 29$ & 0.734145 & 0.944081 & 0.220953 & $0.041 *$ \\
\hline $\mathrm{C} 24$ & $0.9408(2)$ & $0.97115(14)$ & $0.21517(16)$ & $0.0355(4)$ \\
\hline H31 & 0.978425 & 1.029887 & 0.269721 & $0.043 *$ \\
\hline
\end{tabular}




\begin{tabular}{|c|c|c|c|c|}
\hline H32 & 0.988391 & 0.975263 & 0.165338 & $0.043 *$ \\
\hline $\mathrm{C} 25$ & $1.03702(19)$ & $0.59030(12)$ & $0.20584(12)$ & $0.0248(3)$ \\
\hline H33 & 1.054854 & 0.660915 & 0.237426 & $0.030^{*}$ \\
\hline $\mathrm{C} 26$ & $0.9230(2)$ & $0.52439(15)$ & $0.23685(15)$ & $0.0338(4)$ \\
\hline H34 & 0.840624 & 0.544594 & 0.221742 & $0.041 *$ \\
\hline H35 & 0.897781 & 0.454947 & 0.200854 & $0.041 *$ \\
\hline $\mathrm{C} 27$ & $0.9664(2)$ & $0.53054(16)$ & $0.34234(15)$ & $0.0401(5)$ \\
\hline H36 & 0.983959 & 0.598669 & 0.378675 & $0.048^{*}$ \\
\hline H37 & 0.890966 & 0.485109 & 0.358858 & $0.048 *$ \\
\hline $\mathrm{C} 28$ & $1.0950(3)$ & $0.50317(16)$ & $0.36923(15)$ & $0.0405(5)$ \\
\hline H39 & 1.075296 & 0.433155 & 0.337186 & $0.049 *$ \\
\hline H38 & 1.123447 & 0.510441 & 0.438347 & $0.049 *$ \\
\hline $\mathrm{C} 29$ & $1.2107(2)$ & $0.56912(18)$ & $0.34099(15)$ & $0.0417(5)$ \\
\hline H41 & 1.291641 & 0.547196 & 0.355210 & $0.050^{*}$ \\
\hline $\mathrm{H} 40$ & 1.237522 & 0.637984 & 0.378925 & $0.050 *$ \\
\hline $\mathrm{C} 30$ & $1.1689(2)$ & $0.56665(15)$ & $0.23579(14)$ & $0.0324(4)$ \\
\hline $\mathrm{H} 42$ & 1.244177 & 0.615136 & 0.221918 & $0.039 *$ \\
\hline $\mathrm{H} 43$ & 1.156115 & 0.500118 & 0.198041 & $0.039 *$ \\
\hline C31 & $0.8585(2)$ & $0.70279(15)$ & $-0.06282(14)$ & $0.0359(4)$ \\
\hline $\mathrm{H} 44$ & 0.882137 & 0.765597 & -0.013568 & $0.043^{*}$ \\
\hline $\mathrm{C} 32$ & $0.7040(3)$ & $0.6540(2)$ & $-0.0967(2)$ & $0.0536(6)$ \\
\hline H45 & 0.669609 & 0.640626 & -0.042284 & $0.064^{*}$ \\
\hline H46 & 0.680299 & 0.589873 & -0.142688 & $0.064 *$ \\
\hline C33 & $0.6331(4)$ & $0.7193(3)$ & $-0.1434(3)$ & $0.0720(9)$ \\
\hline $\mathrm{H} 48$ & 0.532751 & 0.683588 & -0.167797 & $0.086^{*}$ \\
\hline $\mathrm{H} 47$ & 0.648538 & 0.780594 & -0.095703 & $0.086^{*}$ \\
\hline $\mathrm{C} 34$ & $0.6882(4)$ & $0.7455(3)$ & $-0.2231(2)$ & $0.0704(9)$ \\
\hline $\mathrm{H} 50$ & 0.661290 & 0.684754 & -0.274381 & $0.084^{*}$ \\
\hline H49 & 0.646067 & 0.791608 & -0.248707 & $0.084^{*}$ \\
\hline $\mathrm{C} 35$ & $0.8414(3)$ & $0.7924(2)$ & $-0.1931(2)$ & $0.0610(7)$ \\
\hline H51 & 0.867929 & 0.857776 & -0.148375 & $0.073^{*}$ \\
\hline H52 & 0.873055 & 0.803077 & -0.249146 & $0.073 *$ \\
\hline C36 & $0.9122(3)$ & $0.72721(18)$ & $-0.14558(17)$ & $0.0465(5)$ \\
\hline H54 & 0.895094 & 0.665048 & -0.192628 & $0.056^{*}$ \\
\hline H53 & 1.012784 & 0.762288 & -0.122444 & $0.056^{*}$ \\
\hline $\mathrm{C} 37$ & $0.6472(3)$ & $0.4020(2)$ & $-0.0033(3)$ & $0.0705(8)$ \\
\hline H55 & 0.680375 & 0.468615 & -0.014340 & $0.085^{*}$ \\
\hline H56 & 0.613828 & 0.407387 & 0.053486 & $0.085^{*}$ \\
\hline C38 & $0.5371(4)$ & $0.3329(4)$ & $-0.0867(3)$ & $0.0974(13)$ \\
\hline H58 & 0.445435 & 0.330165 & -0.079680 & $0.117^{*}$ \\
\hline H57 & 0.546322 & 0.354115 & -0.145022 & $0.117^{*}$ \\
\hline C39 & $0.5549(4)$ & $0.2359(3)$ & -0.0907 (3) & $0.1037(14)$ \\
\hline H60 & 0.505404 & 0.199991 & -0.051531 & $0.124 *$ \\
\hline H59 & 0.522228 & 0.194648 & -0.156541 & $0.124^{*}$ \\
\hline $\mathrm{C} 40$ & $0.7025(4)$ & $0.2623(2)$ & $-0.0526(3)$ & $0.0784(10)$ \\
\hline H62 & 0.723819 & 0.214845 & -0.016809 & $0.094 *$ \\
\hline H61 & 0.746114 & 0.260588 & -0.104635 & $0.094 *$ \\
\hline C41 & $0.5148(4)$ & $0.9502(2)$ & -0.08088 (19) & $0.0607(8)$ \\
\hline
\end{tabular}




\begin{tabular}{|c|c|c|c|c|c|}
\hline H65 & 0.523425 & 0.914834 & -0.137403 & $0.073^{*}$ & \\
\hline $\mathrm{C} 42 \mathrm{~A}$ & $0.6311(4)$ & $1.0072(2)$ & $-0.00946(18)$ & $0.0573(7)$ & 0.5 \\
\hline $\mathrm{C} 42 \mathrm{~B}$ & $0.6311(4)$ & $1.0072(2)$ & $-0.00946(18)$ & $0.0573(7)$ & 0.5 \\
\hline H64 & 0.719917 & 1.012313 & -0.016020 & $0.069^{*}$ & 0.5 \\
\hline $\mathrm{C} 43$ & $0.6154(4)$ & $1.0569(2)$ & 0.07242 (19) & $0.0604(7)$ & \\
\hline H63 & 0.694287 & 1.096225 & 0.123303 & $0.072 *$ & \\
\hline $\mathrm{C} 44$ & $0.7893(6)$ & $1.0222(4)$ & $-0.0176(4)$ & $0.0552(13)$ & 0.5 \\
\hline H67 & 0.851023 & 1.031026 & 0.044882 & $0.066^{*}$ & 0.5 \\
\hline H68 & 0.821343 & 1.080825 & -0.041261 & $0.066^{*}$ & 0.5 \\
\hline H66 & 0.788893 & 0.963722 & -0.061378 & $0.066^{*}$ & 0.5 \\
\hline N1 & $0.77781(15)$ & $0.87526(10)$ & $0.44791(10)$ & $0.0232(3)$ & \\
\hline N2 & $0.80123(15)$ & $1.03151(10)$ & $0.41855(10)$ & $0.0228(3)$ & \\
\hline N3 & $0.99709(17)$ & $0.57779(11)$ & $0.10369(10)$ & $0.0262(3)$ & \\
\hline N4 & $0.9242(2)$ & $0.63837(12)$ & $-0.02199(11)$ & $0.0335(4)$ & \\
\hline $\mathrm{AG}$ & $0.98689(2)$ & $0.91010(2)$ & $0.51539(2)$ & $0.02283(5)$ & \\
\hline LI & $0.9428(4)$ & $0.4339(2)$ & $0.0324(3)$ & $0.0376(8)$ & \\
\hline $\mathrm{O}$ & $0.75344(18)$ & $0.35921(12)$ & $0.00793(13)$ & $0.0502(4)$ & \\
\hline
\end{tabular}

Atomic displacement parameters $\left(\AA^{2}\right)$

\begin{tabular}{|c|c|c|c|c|c|c|}
\hline & $U^{11}$ & $U^{22}$ & $U^{33}$ & $U^{12}$ & $U^{13}$ & $U^{23}$ \\
\hline $\mathrm{C} 1$ & $0.0194(8)$ & $0.0237(8)$ & $0.0225(8)$ & $0.0091(6)$ & $0.0054(6)$ & $0.0037(6)$ \\
\hline $\mathrm{C} 2$ & $0.0249(9)$ & $0.0215(8)$ & $0.0282(8)$ & $0.0090(7)$ & $0.0071(7)$ & $0.0068(7)$ \\
\hline $\mathrm{C} 3$ & $0.0243(9)$ & $0.0273(8)$ & $0.0339(9)$ & $0.0084(7)$ & $0.0072(7)$ & $0.0078(7)$ \\
\hline $\mathrm{C} 4$ & $0.0201(9)$ & $0.0420(11)$ & $0.0444(12)$ & $0.0061(8)$ & $0.0043(8)$ & $0.0117(9)$ \\
\hline $\mathrm{C} 5$ & $0.0333(12)$ & $0.0762(17)$ & $0.0590(15)$ & $0.0316(12)$ & $0.0130(11)$ & $0.0194(13)$ \\
\hline C6 & $0.0328(12)$ & 0.0764 (17) & $0.0522(14)$ & $0.0245(12)$ & $0.0060(10)$ & $0.0296(13)$ \\
\hline $\mathrm{C} 7$ & $0.0216(8)$ & $0.0224(8)$ & $0.0331(9)$ & $0.0071(7)$ & $0.0096(7)$ & $0.0103(7)$ \\
\hline $\mathrm{C} 8$ & $0.0399(11)$ & $0.0230(9)$ & $0.0437(11)$ & $0.0020(8)$ & $0.0210(9)$ & $0.0042(8)$ \\
\hline C9 & $0.0569(15)$ & $0.0252(10)$ & 0.0702 (17) & $-0.0004(10)$ & $0.0374(13)$ & $-0.0006(10)$ \\
\hline C10 & $0.0546(15)$ & $0.0255(10)$ & $0.104(2)$ & $0.0185(10)$ & $0.0504(15)$ & $0.0257(12)$ \\
\hline $\mathrm{C} 11$ & $0.0429(13)$ & $0.0381(11)$ & $0.0662(15)$ & $0.0175(10)$ & $0.0275(11)$ & $0.0327(11)$ \\
\hline $\mathrm{C} 12$ & 0.0337 (11) & $0.0366(10)$ & $0.0427(11)$ & $0.0111(9)$ & $0.0129(9)$ & $0.0206(9)$ \\
\hline C13 & $0.0212(8)$ & $0.0217(8)$ & $0.0249(8)$ & $0.0100(7)$ & $0.0025(6)$ & $0.0045(6)$ \\
\hline $\mathrm{C} 14$ & $0.0336(10)$ & $0.0322(9)$ & $0.0304(9)$ & $0.0182(8)$ & $0.0102(8)$ & $0.0066(7)$ \\
\hline $\mathrm{C} 15$ & $0.0431(12)$ & $0.0388(11)$ & $0.0472(12)$ & $0.0278(10)$ & $0.0158(10)$ & $0.0080(9)$ \\
\hline $\mathrm{C} 16$ & $0.0504(13)$ & $0.0281(9)$ & $0.0447(12)$ & $0.0238(9)$ & $0.0084(10)$ & $0.0066(8)$ \\
\hline $\mathrm{C} 17$ & $0.0446(12)$ & $0.0247(9)$ & $0.0381(10)$ & $0.0161(8)$ & $0.0089(9)$ & $0.0104(8)$ \\
\hline C18 & $0.0317(10)$ & $0.0237(8)$ & $0.0372(10)$ & $0.0131(7)$ & $0.0115(8)$ & $0.0106(7)$ \\
\hline C19 & $0.0282(9)$ & $0.0203(8)$ & $0.0275(9)$ & $0.0087(7)$ & $0.0095(7)$ & $0.0024(7)$ \\
\hline $\mathrm{C} 20$ & $0.0314(10)$ & $0.0227(8)$ & $0.0265(8)$ & $0.0105(7)$ & $0.0099(7)$ & $0.0074(7)$ \\
\hline $\mathrm{C} 21$ & $0.0274(9)$ & $0.0234(8)$ & $0.0273(9)$ & $0.0090(7)$ & $0.0096(7)$ & $0.0072(7)$ \\
\hline $\mathrm{C} 22$ & $0.0310(9)$ & $0.0234(8)$ & $0.0253(8)$ & $0.0112(7)$ & $0.0117(7)$ & $0.0031(7)$ \\
\hline $\mathrm{C} 23$ & $0.0333(10)$ & $0.0332(10)$ & $0.0404(11)$ & $0.0183(8)$ & $0.0125(9)$ & $0.0050(8)$ \\
\hline $\mathrm{C} 24$ & $0.0366(11)$ & $0.0224(8)$ & $0.0512(12)$ & $0.0110(8)$ & $0.0192(9)$ & $0.0080(8)$ \\
\hline $\mathrm{C} 25$ & $0.0300(9)$ & $0.0204(8)$ & $0.0236(8)$ & $0.0091(7)$ & $0.0085(7)$ & $0.0022(6)$ \\
\hline $\mathrm{C} 26$ & $0.0302(10)$ & $0.0368(10)$ & $0.0374(10)$ & $0.0110(8)$ & $0.0146(8)$ & $0.0107(8)$ \\
\hline $\mathrm{C} 27$ & 0.0507 (13) & $0.0404(11)$ & $0.0387(11)$ & $0.0178(10)$ & $0.0248(10)$ & $0.0140(9)$ \\
\hline
\end{tabular}




\begin{tabular}{lllllll} 
C28 & $0.0592(14)$ & $0.0392(11)$ & $0.0284(10)$ & $0.0218(10)$ & $0.0138(10)$ & $0.0110(8)$ \\
C29 & $0.0391(12)$ & $0.0524(13)$ & $0.0327(10)$ & $0.0189(10)$ & $0.0044(9)$ & $0.0098(9)$ \\
C30 & $0.0301(10)$ & $0.0401(10)$ & $0.0289(9)$ & $0.0143(8)$ & $0.0096(8)$ & $0.0078(8)$ \\
C31 & $0.0546(13)$ & $0.0294(9)$ & $0.0297(9)$ & $0.0227(9)$ & $0.0119(9)$ & $0.0084(8)$ \\
C32 & $0.0553(16)$ & $0.0652(16)$ & $0.0641(16)$ & $0.0338(13)$ & $0.0295(13)$ & $0.0382(14)$ \\
C33 & $0.068(2)$ & $0.096(2)$ & $0.085(2)$ & $0.0532(19)$ & $0.0291(17)$ & $0.0537(19)$ \\
C34 & $0.077(2)$ & $0.088(2)$ & $0.0618(17)$ & $0.0413(19)$ & $0.0155(16)$ & $0.0424(17)$ \\
C35 & $0.084(2)$ & $0.0543(15)$ & $0.0544(15)$ & $0.0230(15)$ & $0.0245(15)$ & $0.0324(13)$ \\
C36 & $0.0538(15)$ & $0.0424(12)$ & $0.0468(13)$ & $0.0146(11)$ & $0.0181(11)$ & $0.0183(10)$ \\
C37 & $0.0471(16)$ & $0.0643(18)$ & $0.095(2)$ & $0.0133(14)$ & $0.0204(16)$ & $0.0159(17)$ \\
C38 & $0.0507(19)$ & $0.154(4)$ & $0.078(2)$ & $0.026(2)$ & $0.0113(17)$ & $0.028(2)$ \\
C39 & $0.059(2)$ & $0.096(3)$ & $0.104(3)$ & $-0.016(2)$ & $0.014(2)$ & $-0.021(2)$ \\
C40 & $0.070(2)$ & $0.0409(14)$ & $0.098(2)$ & $-0.0083(14)$ & $0.0217(18)$ & $-0.0008(15)$ \\
C41 & $0.100(2)$ & $0.0539(15)$ & $0.0398(13)$ & $0.0408(16)$ & $0.0238(15)$ & $0.0118(11)$ \\
C42A & $0.092(2)$ & $0.0514(14)$ & $0.0405(13)$ & $0.0388(15)$ & $0.0191(14)$ & $0.0177(11)$ \\
C42B & $0.092(2)$ & $0.0514(14)$ & $0.0405(13)$ & $0.0388(15)$ & $0.0191(14)$ & $0.0177(11)$ \\
C43 & $0.090(2)$ & $0.0562(15)$ & $0.0389(13)$ & $0.0335(15)$ & $0.0151(14)$ & $0.0113(11)$ \\
C44 & $0.059(3)$ & $0.068(3)$ & $0.045(3)$ & $0.026(3)$ & $0.017(2)$ & $0.021(2)$ \\
N1 & $0.0202(7)$ & $0.0207(7)$ & $0.0289(7)$ & $0.0077(6)$ & $0.0051(6)$ & $0.0073(6)$ \\
N2 & $0.0196(7)$ & $0.0214(7)$ & $0.0281(7)$ & $0.0098(6)$ & $0.0036(6)$ & $0.0070(6)$ \\
N3 & $0.0341(8)$ & $0.0220(7)$ & $0.0235(7)$ & $0.0120(6)$ & $0.0087(6)$ & $0.0031(6)$ \\
N4 & $0.0515(11)$ & $0.0293(8)$ & $0.0265(8)$ & $0.0233(8)$ & $0.0118(7)$ & $0.0069(6)$ \\
AG & $0.01875(7)$ & $0.02032(7)$ & $0.02912(8)$ & $0.00771(5)$ & $0.00391(5)$ & $0.00737(5)$ \\
LI & $0.042(2)$ & $0.0290(16)$ & $0.0412(19)$ & $0.0127(15)$ & $0.0145(16)$ & $0.0037(14)$ \\
O & $0.0445(10)$ & $0.0380(8)$ & $0.0559(10)$ & $0.0024(7)$ & $0.0143(8)$ & $0.0005(7)$ \\
& & & & & & \\
\hline
\end{tabular}

Geometric parameters $\left(\AA,{ }^{\circ}\right)$

\begin{tabular}{llll}
\hline $\mathrm{C} 1-\mathrm{N} 1$ & $1.329(2)$ & $\mathrm{C} 26-\mathrm{H} 35$ & 0.9900 \\
$\mathrm{C} 1-\mathrm{N} 2$ & $1.331(2)$ & $\mathrm{C} 27-\mathrm{C} 28$ & $1.515(3)$ \\
$\mathrm{C} 1-\mathrm{C} 2$ & $1.450(2)$ & $\mathrm{C} 27-\mathrm{H} 36$ & 0.9900 \\
$\mathrm{C} 2-\mathrm{C} 3$ & $1.194(3)$ & $\mathrm{C} 27-\mathrm{H} 37$ & 0.9900 \\
$\mathrm{C} 3-\mathrm{C} 4$ & $1.433(3)$ & $\mathrm{C} 28-\mathrm{C} 29$ & $1.517(3)$ \\
$\mathrm{C} 4-\mathrm{C} 5$ & $1.501(3)$ & $\mathrm{C} 28-\mathrm{H} 39$ & 0.9900 \\
$\mathrm{C} 4-\mathrm{C} 6$ & $1.505(3)$ & $\mathrm{C} 28-\mathrm{H} 38$ & 0.9900 \\
$\mathrm{C} 4-\mathrm{H} 1$ & $\mathrm{C} 29-\mathrm{C} 30$ & $1.531(3)$ \\
$\mathrm{C} 5-\mathrm{C} 6$ & 1.0000 & $\mathrm{C} 29-\mathrm{H} 41$ & 0.9900 \\
$\mathrm{C} 5-\mathrm{H} 3$ & $1.473(4)$ & $\mathrm{C} 29-\mathrm{H} 40$ & 0.9900 \\
$\mathrm{C} 5-\mathrm{H} 2$ & 0.9900 & $\mathrm{C} 30-\mathrm{H} 42$ & 0.9900 \\
$\mathrm{C} 6-\mathrm{H} 4$ & 0.9900 & $\mathrm{C} 30-\mathrm{H} 43$ & 0.9900 \\
$\mathrm{C} 6-\mathrm{H} 5$ & 0.9900 & $\mathrm{C} 31-\mathrm{N} 4$ & $1.455(2)$ \\
$\mathrm{C} 7-\mathrm{N} 1$ & 0.9900 & $\mathrm{C} 31-\mathrm{C} 32$ & $1.512(4)$ \\
$\mathrm{C} 7-\mathrm{C} 8$ & $1.467(2)$ & $\mathrm{C} 31-\mathrm{C} 36$ & $1.529(3)$ \\
$\mathrm{C} 7-\mathrm{C} 12$ & $1.520(3)$ & $\mathrm{C} 31-\mathrm{H} 44$ & 1.0000 \\
$\mathrm{C} 7-\mathrm{H} 6$ & $1.526(3)$ & $\mathrm{C} 32-\mathrm{C} 33$ & $1.536(3)$ \\
$\mathrm{C} 8-\mathrm{C} 9$ & 1.0000 & $\mathrm{C} 32-\mathrm{H} 45$ & 0.9900 \\
$\mathrm{C} 8-\mathrm{H} 86$ & $\mathrm{C} 33-\mathrm{C} 34$ & 0.9900 \\
$\mathrm{C} 8-\mathrm{H} 7$ & $1.533(3)$ & & $1.501(4)$
\end{tabular}




\begin{tabular}{|c|c|c|c|}
\hline $\mathrm{C} 9-\mathrm{C} 10$ & $1.506(4)$ & $\mathrm{C} 33-\mathrm{H} 48$ & 0.9900 \\
\hline C9-H9 & 0.9900 & $\mathrm{C} 33-\mathrm{H} 47$ & 0.9900 \\
\hline C9- $\mathrm{H} 10$ & 0.9900 & $\mathrm{C} 34-\mathrm{C} 35$ & $1.497(5)$ \\
\hline $\mathrm{C} 10-\mathrm{C} 11$ & $1.516(4)$ & $\mathrm{C} 34-\mathrm{H} 50$ & 0.9900 \\
\hline $\mathrm{C} 10-\mathrm{H} 12$ & 0.9900 & C34-H49 & 0.9900 \\
\hline $\mathrm{C} 10-\mathrm{H} 11$ & 0.9900 & $\mathrm{C} 35-\mathrm{C} 36$ & $1.540(4)$ \\
\hline $\mathrm{C} 11-\mathrm{C} 12$ & $1.526(3)$ & C35-H51 & 0.9900 \\
\hline $\mathrm{C} 11-\mathrm{H} 13$ & 0.9900 & C $35-\mathrm{H} 52$ & 0.9900 \\
\hline $\mathrm{C} 11-\mathrm{H} 14$ & 0.9900 & C36-H54 & 0.9900 \\
\hline C12-H16 & 0.9900 & $\mathrm{C} 36-\mathrm{H} 53$ & 0.9900 \\
\hline $\mathrm{C} 12-\mathrm{H} 15$ & 0.9900 & $\mathrm{C} 37-\mathrm{O}$ & $1.426(4)$ \\
\hline $\mathrm{C} 13-\mathrm{N} 2$ & $1.472(2)$ & C37-C38 & $1.479(5)$ \\
\hline $\mathrm{C} 13-\mathrm{C} 18$ & $1.521(3)$ & C $37-\mathrm{H} 55$ & 0.9900 \\
\hline $\mathrm{C} 13-\mathrm{C} 14$ & $1.521(2)$ & C37-H56 & 0.9900 \\
\hline $\mathrm{C} 13-\mathrm{H} 17$ & 1.0000 & $\mathrm{C} 38-\mathrm{C} 39$ & $1.470(6)$ \\
\hline $\mathrm{C} 14-\mathrm{C} 15$ & 1.530 & C $38-\mathrm{H} 58$ & 0.9900 \\
\hline C14-H18 & 0.9900 & C38-H57 & 0.9900 \\
\hline C14-H19 & 0.9900 & $\mathrm{C} 39-\mathrm{C} 40$ & $1.450(5)$ \\
\hline $\mathrm{C} 15-\mathrm{C} 16$ & 1.513 & $\mathrm{C} 39-\mathrm{H} 60$ & 0.9900 \\
\hline $\mathrm{C} 15-\mathrm{H} 21$ & 0.9900 & C39-H59 & 0.9900 \\
\hline $\mathrm{C} 15-\mathrm{H} 20$ & 0.9900 & $\mathrm{C} 40-\mathrm{O}$ & $1.419(3)$ \\
\hline $\mathrm{C} 16-\mathrm{C} 17$ & $1.522(3)$ & C40-H62 & 0.9900 \\
\hline $\mathrm{C} 16-\mathrm{H} 22$ & 0.9900 & $\mathrm{C} 40-\mathrm{H} 61$ & 0.9900 \\
\hline $\mathrm{C} 16-\mathrm{H} 23$ & 0.9900 & $\mathrm{C} 41-\mathrm{C} 42 \mathrm{~B}$ & $1.371(4)$ \\
\hline $\mathrm{C} 17-\mathrm{C} 18$ & 1.529 (2) & $\mathrm{C} 41-\mathrm{C} 42 \mathrm{~A}$ & $1.371(4)$ \\
\hline C17-H25 & 0.9900 & $\mathrm{C} 41-\mathrm{C} 43^{\mathrm{ii}}$ & $1.388(5)$ \\
\hline $\mathrm{C} 17-\mathrm{H} 24$ & 0.9900 & $\mathrm{C} 41-\mathrm{H} 65$ & 0.9500 \\
\hline $\mathrm{C} 18-\mathrm{H} 27$ & 0.9900 & $\mathrm{C} 42 \mathrm{~A}-\mathrm{C} 43$ & $1.383(4)$ \\
\hline $\mathrm{C} 18-\mathrm{H} 26$ & 0.9900 & $\mathrm{C} 42 \mathrm{~A}-\mathrm{C} 44$ & $1.661(6)$ \\
\hline $\mathrm{C} 19-\mathrm{N} 4$ & $1.321(2)$ & $\mathrm{C} 42 \mathrm{~B}-\mathrm{C} 43$ & $1.383(4)$ \\
\hline $\mathrm{C} 19-\mathrm{N} 3$ & $1.335(2)$ & $\mathrm{C} 42 \mathrm{~B}-\mathrm{H} 64$ & 0.9500 \\
\hline $\mathrm{C} 19-\mathrm{C} 20$ & $1.466(2)$ & $\mathrm{C} 43-\mathrm{C} 41^{\mathrm{ii}}$ & $1.388(5)$ \\
\hline $\mathrm{C} 19-\mathrm{LI}^{\mathrm{i}}$ & $2.428(4)$ & $\mathrm{C} 43-\mathrm{H} 63$ & 0.9500 \\
\hline $\mathrm{C} 20-\mathrm{C} 21$ & $1.189(3)$ & $\mathrm{C} 44-\mathrm{H} 67$ & 0.9800 \\
\hline $\mathrm{C} 21-\mathrm{C} 22$ & $1.437(2)$ & $\mathrm{C} 44-\mathrm{H} 68$ & 0.9800 \\
\hline $\mathrm{C} 22-\mathrm{C} 23$ & $1.503(3)$ & $\mathrm{C} 44-\mathrm{H} 66$ & 0.9800 \\
\hline $\mathrm{C} 22-\mathrm{C} 24$ & 1.507 (3) & $\mathrm{N} 1-\mathrm{AG}$ & 2.0908 \\
\hline $\mathrm{C} 22-\mathrm{H} 28$ & 1.0000 & $\mathrm{~N} 2-\mathrm{AG}^{\mathrm{iii}}$ & $2.0916(14)$ \\
\hline $\mathrm{C} 23-\mathrm{C} 24$ & $1.486(3)$ & N3-LI & $2.033(4)$ \\
\hline $\mathrm{C} 23-\mathrm{H} 30$ & 0.9900 & $\mathrm{~N} 3-\mathrm{LI}^{\mathrm{i}}$ & $2.261(4)$ \\
\hline $\mathrm{C} 23-\mathrm{H} 29$ & 0.9900 & $\mathrm{~N} 4-\mathrm{LI}^{\mathrm{i}}$ & $2.001(4)$ \\
\hline $\mathrm{C} 24-\mathrm{H} 31$ & 0.9900 & $\mathrm{AG}-\mathrm{N} 2^{\mathrm{iii}}$ & $2.0917(15)$ \\
\hline $\mathrm{C} 24-\mathrm{H} 32$ & 0.9900 & $\mathrm{AG}-\mathrm{AG}^{\mathrm{iii}}$ & 2.6838 \\
\hline $\mathrm{C} 25-\mathrm{N} 3$ & 1.459 (2) & $\mathrm{LI}-\mathrm{O}$ & $1.906(4)$ \\
\hline $\mathrm{C} 25-\mathrm{C} 30$ & $1.523(3)$ & $\mathrm{LI}-\mathrm{N} 4^{\mathrm{i}}$ & $2.001(4)$ \\
\hline $\mathrm{C} 25-\mathrm{C} 26$ & $1.528(3)$ & $\mathrm{LI}-\mathrm{N} 3^{\mathrm{i}}$ & $2.261(4)$ \\
\hline $\mathrm{C} 25-\mathrm{H} 33$ & 1.0000 & $\mathrm{LI}-\mathrm{C} 19^{\mathrm{i}}$ & $2.428(4)$ \\
\hline $\mathrm{C} 26-\mathrm{C} 27$ & $1.524(3)$ & $\mathrm{LI}-\mathrm{LI}^{\mathrm{i}}$ & $2.440(7)$ \\
\hline
\end{tabular}


C26-H34

$\mathrm{N} 1-\mathrm{C} 1-\mathrm{N} 2$

$\mathrm{N} 1-\mathrm{C} 1-\mathrm{C} 2$

$\mathrm{N} 2-\mathrm{C} 1-\mathrm{C} 2$

$\mathrm{C} 3-\mathrm{C} 2-\mathrm{C} 1$

$\mathrm{C} 2-\mathrm{C} 3-\mathrm{C} 4$

$\mathrm{C} 3-\mathrm{C} 4-\mathrm{C} 5$

$\mathrm{C} 3-\mathrm{C} 4-\mathrm{C} 6$

$\mathrm{C} 5-\mathrm{C} 4-\mathrm{C} 6$

$\mathrm{C} 3-\mathrm{C} 4-\mathrm{H} 1$

$\mathrm{C} 5-\mathrm{C} 4-\mathrm{H} 1$

$\mathrm{C} 6-\mathrm{C} 4-\mathrm{H} 1$

$\mathrm{C} 6-\mathrm{C} 5-\mathrm{C} 4$

$\mathrm{C} 6-\mathrm{C} 5-\mathrm{H} 3$

$\mathrm{C} 4-\mathrm{C} 5-\mathrm{H} 3$

$\mathrm{C} 6-\mathrm{C} 5-\mathrm{H} 2$

$\mathrm{C} 4-\mathrm{C} 5-\mathrm{H} 2$

$\mathrm{H} 3-\mathrm{C} 5-\mathrm{H} 2$

$\mathrm{C} 5-\mathrm{C} 6-\mathrm{C} 4$

$\mathrm{C} 5-\mathrm{C} 6-\mathrm{H} 4$

$\mathrm{C} 4-\mathrm{C} 6-\mathrm{H} 4$

$\mathrm{C} 5-\mathrm{C} 6-\mathrm{H} 5$

$\mathrm{C} 4-\mathrm{C} 6-\mathrm{H} 5$

$\mathrm{H} 4-\mathrm{C} 6-\mathrm{H} 5$

$\mathrm{N} 1-\mathrm{C} 7-\mathrm{C} 8$

$\mathrm{N} 1-\mathrm{C} 7-\mathrm{C} 12$

$\mathrm{C} 8-\mathrm{C} 7-\mathrm{C} 12$

$\mathrm{N} 1-\mathrm{C} 7-\mathrm{H} 6$

$\mathrm{C} 8-\mathrm{C} 7-\mathrm{H} 6$

$\mathrm{C} 12-\mathrm{C} 7-\mathrm{H} 6$

$\mathrm{C} 7-\mathrm{C} 8-\mathrm{C} 9$

$\mathrm{C} 7-\mathrm{C} 8-\mathrm{H} 8$

$\mathrm{C} 9-\mathrm{C} 8-\mathrm{H} 8$

$\mathrm{C} 7-\mathrm{C} 8-\mathrm{H} 7$

C9- 8 - $-\mathrm{H} 7$

$\mathrm{H} 8-\mathrm{C} 8-\mathrm{H} 7$

$\mathrm{C} 10-\mathrm{C} 9-\mathrm{C} 8$

$\mathrm{C} 10-\mathrm{C} 9-\mathrm{H} 9$

C $8-\mathrm{C} 9-\mathrm{H} 9$

$\mathrm{C} 10-\mathrm{C} 9-\mathrm{H} 10$

C8- $99-\mathrm{H} 10$

$\mathrm{H} 9-\mathrm{C} 9-\mathrm{H} 10$

C9- $\mathrm{C} 10-\mathrm{C} 11$

C9- $110-\mathrm{H} 12$

$\mathrm{C} 11-\mathrm{C} 10-\mathrm{H} 12$

C9- $10-\mathrm{H} 11$

$\mathrm{C} 11-\mathrm{C} 10-\mathrm{H} 11$

0.9900

$123.71(15)$

$118.48(15)$

$117.81(15)$

$175.75(19)$

$176.6(2)$

$118.31(19)$

$118.78(19)$

58.69 (17)

116.3

116.3

116.3

60.81 (16)

117.7

117.7

117.7

117.7

114.8

$60.50(16)$

117.7

117.7

117.7

117.7

114.8

$110.92(14)$

$110.30(15)$

110.42 (16)

108.4

108.4

108.4

111.51 (17)

109.3

109.3

109.3

109.3

108.0

$111.6(2)$

109.3

109.3

109.3

109.3

108.0

$111.62(18)$

109.3

109.3

109.3

109.3
$\mathrm{C} 26-\mathrm{C} 27-\mathrm{H} 37$

$\mathrm{H} 36-\mathrm{C} 27-\mathrm{H} 37$

C27-C28-C29

C27-C28-H39

C29-C28-H39

$\mathrm{C} 27-\mathrm{C} 28-\mathrm{H} 38$

$\mathrm{C} 29-\mathrm{C} 28-\mathrm{H} 38$

$\mathrm{H} 39-\mathrm{C} 28-\mathrm{H} 38$

$\mathrm{C} 28-\mathrm{C} 29-\mathrm{C} 30$

$\mathrm{C} 28-\mathrm{C} 29-\mathrm{H} 41$

$\mathrm{C} 30-\mathrm{C} 29-\mathrm{H} 41$

$\mathrm{C} 28-\mathrm{C} 29-\mathrm{H} 40$

$\mathrm{C} 30-\mathrm{C} 29-\mathrm{H} 40$

$\mathrm{H} 41-\mathrm{C} 29-\mathrm{H} 40$

$\mathrm{C} 25-\mathrm{C} 30-\mathrm{C} 29$

$\mathrm{C} 25-\mathrm{C} 30-\mathrm{H} 42$

$\mathrm{C} 29-\mathrm{C} 30-\mathrm{H} 42$

$\mathrm{C} 25-\mathrm{C} 30-\mathrm{H} 43$

$\mathrm{C} 29-\mathrm{C} 30-\mathrm{H} 43$

$\mathrm{H} 42-\mathrm{C} 30-\mathrm{H} 43$

$\mathrm{N} 4-\mathrm{C} 31-\mathrm{C} 32$

$\mathrm{N} 4-\mathrm{C} 31-\mathrm{C} 36$

$\mathrm{C} 32-\mathrm{C} 31-\mathrm{C} 36$

$\mathrm{N} 4-\mathrm{C} 31-\mathrm{H} 44$

C32-C $31-\mathrm{H} 44$

C36- C31-H44

C31-C32-C33

$\mathrm{C} 31-\mathrm{C} 32-\mathrm{H} 45$

$\mathrm{C} 33-\mathrm{C} 32-\mathrm{H} 45$

$\mathrm{C} 31-\mathrm{C} 32-\mathrm{H} 46$

$\mathrm{C} 33-\mathrm{C} 32-\mathrm{H} 46$

$\mathrm{H} 45-\mathrm{C} 32-\mathrm{H} 46$

C34-C33-C32

$\mathrm{C} 34-\mathrm{C} 33-\mathrm{H} 48$

C32- $333-\mathrm{H} 48$

$\mathrm{C} 34-\mathrm{C} 33-\mathrm{H} 47$

C32- $333-\mathrm{H} 47$

$\mathrm{H} 48-\mathrm{C} 33-\mathrm{H} 47$

$\mathrm{C} 35-\mathrm{C} 34-\mathrm{C} 33$

C $35-\mathrm{C} 34-\mathrm{H} 50$

C $33-\mathrm{C} 34-\mathrm{H} 50$

C35-C34- 449

$\mathrm{C} 33-\mathrm{C} 34-\mathrm{H} 49$

$\mathrm{H} 50-\mathrm{C} 34-\mathrm{H} 49$

$\mathrm{C} 34-\mathrm{C} 35-\mathrm{C} 36$

$\mathrm{C} 34-\mathrm{C} 35-\mathrm{H} 51$
109.4

108.0

110.48 (17)

109.6

109.6

109.6

109.6

108.1

111.95 (18)

109.2

109.2

109.2

109.2

107.9

112.68 (16)

109.1

109.1

109.1

109.1

107.8

$110.88(18)$

$109.39(18)$

109.73 (19)

108.9

108.9

108.9

111.7 (2)

109.3

109.3

109.3

109.3

107.9

110.7 (2)

109.5

109.5

109.5

109.5

108.1

112.6 (3)

109.1

109.1

109.1

109.1

107.8

111.3 (2)

109.4 


\begin{tabular}{|c|c|c|c|}
\hline $\mathrm{H} 12-\mathrm{C} 10-\mathrm{H} 11$ & 108.0 & $\mathrm{C} 36-\mathrm{C} 35-\mathrm{H} 51$ & 109.4 \\
\hline $\mathrm{C} 10-\mathrm{C} 11-\mathrm{C} 12$ & $111.61(18)$ & C $34-\mathrm{C} 35-\mathrm{H} 52$ & 109.4 \\
\hline $\mathrm{C} 10-\mathrm{C} 11-\mathrm{H} 13$ & 109.3 & $\mathrm{C} 36-\mathrm{C} 35-\mathrm{H} 52$ & 109.4 \\
\hline $\mathrm{C} 12-\mathrm{C} 11-\mathrm{H} 13$ & 109.3 & $\mathrm{H} 51-\mathrm{C} 35-\mathrm{H} 52$ & 108.0 \\
\hline $\mathrm{C} 10-\mathrm{C} 11-\mathrm{H} 14$ & 109.3 & $\mathrm{C} 31-\mathrm{C} 36-\mathrm{C} 35$ & $111.1(2)$ \\
\hline $\mathrm{C} 12-\mathrm{C} 11-\mathrm{H} 14$ & 109.3 & $\mathrm{C} 31-\mathrm{C} 36-\mathrm{H} 54$ & 109.4 \\
\hline $\mathrm{H} 13-\mathrm{C} 11-\mathrm{H} 14$ & 108.0 & $\mathrm{C} 35-\mathrm{C} 36-\mathrm{H} 54$ & 109.4 \\
\hline $\mathrm{C} 7-\mathrm{C} 12-\mathrm{C} 11$ & $111.45(18)$ & $\mathrm{C} 31-\mathrm{C} 36-\mathrm{H} 53$ & 109.4 \\
\hline $\mathrm{C} 7-\mathrm{C} 12-\mathrm{H} 16$ & 109.3 & $\mathrm{C} 35-\mathrm{C} 36-\mathrm{H} 53$ & 109.4 \\
\hline $\mathrm{C} 11-\mathrm{C} 12-\mathrm{H} 16$ & 109.3 & $\mathrm{H} 54-\mathrm{C} 36-\mathrm{H} 53$ & 108.0 \\
\hline $\mathrm{C} 7-\mathrm{C} 12-\mathrm{H} 15$ & 109.3 & $\mathrm{O}-\mathrm{C} 37-\mathrm{C} 38$ & $104.5(3)$ \\
\hline $\mathrm{C} 11-\mathrm{C} 12-\mathrm{H} 15$ & 109.3 & $\mathrm{O}-\mathrm{C} 37-\mathrm{H} 55$ & 110.8 \\
\hline $\mathrm{H} 16-\mathrm{C} 12-\mathrm{H} 15$ & 108.0 & $\mathrm{C} 38-\mathrm{C} 37-\mathrm{H} 55$ & 110.8 \\
\hline $\mathrm{N} 2-\mathrm{C} 13-\mathrm{C} 18$ & $110.41(14)$ & $\mathrm{O}-\mathrm{C} 37-\mathrm{H} 56$ & 110.8 \\
\hline $\mathrm{N} 2-\mathrm{C} 13-\mathrm{C} 14$ & $111.62(14)$ & C $38-\mathrm{C} 37-\mathrm{H} 56$ & 110.8 \\
\hline $\mathrm{C} 18-\mathrm{C} 13-\mathrm{C} 14$ & $109.78(15)$ & $\mathrm{H} 55-\mathrm{C} 37-\mathrm{H} 56$ & 108.9 \\
\hline N2-C13-H17 & 108.3 & $\mathrm{C} 39-\mathrm{C} 38-\mathrm{C} 37$ & $105.6(3)$ \\
\hline $\mathrm{C} 18-\mathrm{C} 13-\mathrm{H} 17$ & 108.3 & $\mathrm{C} 39-\mathrm{C} 38-\mathrm{H} 58$ & 110.6 \\
\hline $\mathrm{C} 14-\mathrm{C} 13-\mathrm{H} 17$ & 108.3 & C37-C $38-\mathrm{H} 58$ & 110.6 \\
\hline $\mathrm{C} 13-\mathrm{C} 14-\mathrm{C} 15$ & $111.17(16)$ & C39-C38-H57 & 110.6 \\
\hline $\mathrm{C} 13-\mathrm{C} 14-\mathrm{H} 18$ & 109.4 & $\mathrm{C} 37-\mathrm{C} 38-\mathrm{H} 57$ & 110.6 \\
\hline $\mathrm{C} 15-\mathrm{C} 14-\mathrm{H} 18$ & 109.4 & $\mathrm{H} 58-\mathrm{C} 38-\mathrm{H} 57$ & 108.8 \\
\hline $\mathrm{C} 13-\mathrm{C} 14-\mathrm{H} 19$ & 109.4 & $\mathrm{C} 40-\mathrm{C} 39-\mathrm{C} 38$ & $102.6(3)$ \\
\hline $\mathrm{C} 15-\mathrm{C} 14-\mathrm{H} 19$ & 109.4 & $\mathrm{C} 40-\mathrm{C} 39-\mathrm{H} 60$ & 111.3 \\
\hline $\mathrm{H} 18-\mathrm{C} 14-\mathrm{H} 19$ & 108.0 & $\mathrm{C} 38-\mathrm{C} 39-\mathrm{H} 60$ & 111.3 \\
\hline $\mathrm{C} 16-\mathrm{C} 15-\mathrm{C} 14$ & $111.29(18)$ & $\mathrm{C} 40-\mathrm{C} 39-\mathrm{H} 59$ & 111.3 \\
\hline $\mathrm{C} 16-\mathrm{C} 15-\mathrm{H} 21$ & 109.4 & C38-C39-H59 & 111.3 \\
\hline $\mathrm{C} 14-\mathrm{C} 15-\mathrm{H} 21$ & 109.4 & $\mathrm{H} 60-\mathrm{C} 39-\mathrm{H} 59$ & 109.2 \\
\hline $\mathrm{C} 16-\mathrm{C} 15-\mathrm{H} 20$ & 109.4 & $\mathrm{O}-\mathrm{C} 40-\mathrm{C} 39$ & $108.0(3)$ \\
\hline $\mathrm{C} 14-\mathrm{C} 15-\mathrm{H} 20$ & 109.4 & $\mathrm{O}-\mathrm{C} 40-\mathrm{H} 62$ & 110.1 \\
\hline $\mathrm{H} 21-\mathrm{C} 15-\mathrm{H} 20$ & 108.0 & $\mathrm{C} 39-\mathrm{C} 40-\mathrm{H} 62$ & 110.1 \\
\hline $\mathrm{C} 15-\mathrm{C} 16-\mathrm{C} 17$ & $111.32(17)$ & $\mathrm{O}-\mathrm{C} 40-\mathrm{H} 61$ & 110.1 \\
\hline $\mathrm{C} 15-\mathrm{C} 16-\mathrm{H} 22$ & 109.4 & $\mathrm{C} 39-\mathrm{C} 40-\mathrm{H} 61$ & 110.1 \\
\hline $\mathrm{C} 17-\mathrm{C} 16-\mathrm{H} 22$ & 109.4 & $\mathrm{H} 62-\mathrm{C} 40-\mathrm{H} 61$ & 108.4 \\
\hline $\mathrm{C} 15-\mathrm{C} 16-\mathrm{H} 23$ & 109.4 & $\mathrm{C} 42 \mathrm{~B}-\mathrm{C} 41-\mathrm{C} 43^{\mathrm{ii}}$ & $121.4(3)$ \\
\hline $\mathrm{C} 17-\mathrm{C} 16-\mathrm{H} 23$ & 109.4 & $\mathrm{C} 42 \mathrm{~A}-\mathrm{C} 41-\mathrm{C} 43^{\mathrm{ii}}$ & $121.4(3)$ \\
\hline $\mathrm{H} 22-\mathrm{C} 16-\mathrm{H} 23$ & 108.0 & $\mathrm{C} 42 \mathrm{~A}-\mathrm{C} 41-\mathrm{H} 65$ & 119.3 \\
\hline $\mathrm{C} 16-\mathrm{C} 17-\mathrm{C} 18$ & $111.25(17)$ & $\mathrm{C} 43^{\mathrm{ii}}-\mathrm{C} 41-\mathrm{H} 65$ & 119.3 \\
\hline $\mathrm{C} 16-\mathrm{C} 17-\mathrm{H} 25$ & 109.4 & $\mathrm{C} 41-\mathrm{C} 42 \mathrm{~A}-\mathrm{C} 43$ & $118.0(3)$ \\
\hline $\mathrm{C} 18-\mathrm{C} 17-\mathrm{H} 25$ & 109.4 & $\mathrm{C} 41-\mathrm{C} 42 \mathrm{~A}-\mathrm{C} 44$ & $123.4(3)$ \\
\hline $\mathrm{C} 16-\mathrm{C} 17-\mathrm{H} 24$ & 109.4 & $\mathrm{C} 43-\mathrm{C} 42 \mathrm{~A}-\mathrm{C} 44$ & $118.5(3)$ \\
\hline $\mathrm{C} 18-\mathrm{C} 17-\mathrm{H} 24$ & 109.4 & $\mathrm{C} 41-\mathrm{C} 42 \mathrm{~B}-\mathrm{C} 43$ & $118.0(3)$ \\
\hline $\mathrm{H} 25-\mathrm{C} 17-\mathrm{H} 24$ & 108.0 & $\mathrm{C} 41-\mathrm{C} 42 \mathrm{~B}-\mathrm{H} 64$ & 121.0 \\
\hline $\mathrm{C} 13-\mathrm{C} 18-\mathrm{C} 17$ & $111.56(16)$ & $\mathrm{C} 43-\mathrm{C} 42 \mathrm{~B}-\mathrm{H} 64$ & 121.0 \\
\hline $\mathrm{C} 13-\mathrm{C} 18-\mathrm{H} 27$ & 109.3 & $\mathrm{C} 42 \mathrm{~B}-\mathrm{C} 43-\mathrm{C} 41^{\mathrm{ii}}$ & $120.6(3)$ \\
\hline $\mathrm{C} 17-\mathrm{C} 18-\mathrm{H} 27$ & 109.3 & $\mathrm{C} 42 \mathrm{~A}-\mathrm{C} 43-\mathrm{C} 41^{\mathrm{ii}}$ & $120.6(3)$ \\
\hline $\mathrm{C} 13-\mathrm{C} 18-\mathrm{H} 26$ & 109.3 & $\mathrm{C} 42 \mathrm{~A}-\mathrm{C} 43-\mathrm{H} 63$ & 119.7 \\
\hline $\mathrm{C} 17-\mathrm{C} 18-\mathrm{H} 26$ & 109.3 & $\mathrm{C} 41^{\mathrm{ii}}-\mathrm{C} 43-\mathrm{H} 63$ & 119.7 \\
\hline
\end{tabular}




\begin{tabular}{|c|c|c|c|}
\hline $\mathrm{H} 27-\mathrm{C} 18-\mathrm{H} 26$ & 108.0 & $\mathrm{C} 42 \mathrm{~A}-\mathrm{C} 44-\mathrm{H} 67$ & 109.5 \\
\hline N4-C19-N3 & $118.85(16)$ & $\mathrm{C} 42 \mathrm{~A}-\mathrm{C} 44-\mathrm{H} 68$ & 109.5 \\
\hline $\mathrm{N} 4-\mathrm{C} 19-\mathrm{C} 20$ & $120.31(16)$ & $\mathrm{H} 67-\mathrm{C} 44-\mathrm{H} 68$ & 109.5 \\
\hline N3-C19-C20 & $120.77(16)$ & $\mathrm{C} 42 \mathrm{~A}-\mathrm{C} 44-\mathrm{H} 66$ & 109.5 \\
\hline $\mathrm{N} 4-\mathrm{C} 19-\mathrm{LI}^{\mathrm{i}}$ & $55.46(13)$ & $\mathrm{H} 67-\mathrm{C} 44-\mathrm{H} 66$ & 109.5 \\
\hline $\mathrm{N} 3-\mathrm{C} 19-\mathrm{LI}^{\mathrm{i}}$ & $66.70(13)$ & $\mathrm{H} 68-\mathrm{C} 44-\mathrm{H} 66$ & 109.5 \\
\hline $\mathrm{C} 20-\mathrm{C} 19-\mathrm{LI}^{\mathrm{i}}$ & $158.53(15)$ & $\mathrm{C} 1-\mathrm{N} 1-\mathrm{C} 7$ & $119.72(14)$ \\
\hline $\mathrm{C} 21-\mathrm{C} 20-\mathrm{C} 19$ & $176.60(19)$ & $\mathrm{C} 1-\mathrm{N} 1-\mathrm{AG}$ & $122.90(11)$ \\
\hline $\mathrm{C} 20-\mathrm{C} 21-\mathrm{C} 22$ & $177.03(19)$ & $\mathrm{C} 7-\mathrm{N} 1-\mathrm{AG}$ & $117.28(11)$ \\
\hline $\mathrm{C} 21-\mathrm{C} 22-\mathrm{C} 23$ & $118.85(16)$ & $\mathrm{C} 1-\mathrm{N} 2-\mathrm{C} 13$ & $119.09(14)$ \\
\hline $\mathrm{C} 21-\mathrm{C} 22-\mathrm{C} 24$ & $118.48(16)$ & $\mathrm{C} 1-\mathrm{N} 2-\mathrm{AG}^{\mathrm{iii}}$ & $122.58(11)$ \\
\hline $\mathrm{C} 23-\mathrm{C} 22-\mathrm{C} 24$ & $59.15(13)$ & $\mathrm{C} 13-\mathrm{N} 2-\mathrm{AG}^{\mathrm{iii}}$ & $118.05(11)$ \\
\hline $\mathrm{C} 21-\mathrm{C} 22-\mathrm{H} 28$ & 116.1 & $\mathrm{C} 19-\mathrm{N} 3-\mathrm{C} 25$ & $118.58(14)$ \\
\hline $\mathrm{C} 23-\mathrm{C} 22-\mathrm{H} 28$ & 116.1 & C19-N3-LI & $123.43(16)$ \\
\hline $\mathrm{C} 24-\mathrm{C} 22-\mathrm{H} 28$ & 116.1 & $\mathrm{C} 25-\mathrm{N} 3-\mathrm{LI}$ & $111.73(15)$ \\
\hline $\mathrm{C} 24-\mathrm{C} 23-\mathrm{C} 22$ & $60.54(13)$ & $\mathrm{C} 19-\mathrm{N} 3-\mathrm{LI}^{\mathrm{i}}$ & $80.46(14)$ \\
\hline $\mathrm{C} 24-\mathrm{C} 23-\mathrm{H} 30$ & 117.7 & $\mathrm{C} 25-\mathrm{N} 3-\mathrm{LI}^{\mathrm{i}}$ & $148.51(16)$ \\
\hline $\mathrm{C} 22-\mathrm{C} 23-\mathrm{H} 30$ & 117.7 & $\mathrm{LI}-\mathrm{N} 3-\mathrm{LI}^{\mathrm{i}}$ & $69.00(17)$ \\
\hline $\mathrm{C} 24-\mathrm{C} 23-\mathrm{H} 29$ & 117.7 & $\mathrm{C} 19-\mathrm{N} 4-\mathrm{C} 31$ & $119.98(16)$ \\
\hline $\mathrm{C} 22-\mathrm{C} 23-\mathrm{H} 29$ & 117.7 & $\mathrm{C} 19-\mathrm{N} 4-\mathrm{LI}^{\mathrm{i}}$ & $91.58(16)$ \\
\hline $\mathrm{H} 30-\mathrm{C} 23-\mathrm{H} 29$ & 114.8 & $\mathrm{C} 31-\mathrm{N} 4-\mathrm{LI}^{\mathrm{i}}$ & $144.63(18)$ \\
\hline $\mathrm{C} 23-\mathrm{C} 24-\mathrm{C} 22$ & $60.31(13)$ & $\mathrm{N} 1-\mathrm{AG}-\mathrm{N} 2^{\mathrm{iii}}$ & $170.66(5)$ \\
\hline $\mathrm{C} 23-\mathrm{C} 24-\mathrm{H} 31$ & 117.7 & $\mathrm{~N} 1-\mathrm{AG}-\mathrm{AG}^{\mathrm{iii}}$ & $85.27(4)$ \\
\hline $\mathrm{C} 22-\mathrm{C} 24-\mathrm{H} 31$ & 117.7 & $\mathrm{~N} 2^{\mathrm{iii}}-\mathrm{AG}-\mathrm{AG}^{\mathrm{iii}}$ & $85.45(4)$ \\
\hline $\mathrm{C} 23-\mathrm{C} 24-\mathrm{H} 32$ & 117.7 & $\mathrm{O}-\mathrm{LI}-\mathrm{N} 4^{\mathrm{i}}$ & $118.78(19)$ \\
\hline $\mathrm{C} 22-\mathrm{C} 24-\mathrm{H} 32$ & 117.7 & $\mathrm{O}-\mathrm{LI}-\mathrm{N} 3$ & $114.34(19)$ \\
\hline $\mathrm{H} 31-\mathrm{C} 24-\mathrm{H} 32$ & 114.9 & $\mathrm{~N} 4 \mathrm{i}-\mathrm{LI}-\mathrm{N} 3$ & $124.4(2)$ \\
\hline $\mathrm{N} 3-\mathrm{C} 25-\mathrm{C} 30$ & $109.55(15)$ & $\mathrm{O}-\mathrm{LI}-\mathrm{N} 3^{\mathrm{i}}$ & $110.21(18)$ \\
\hline $\mathrm{N} 3-\mathrm{C} 25-\mathrm{C} 26$ & $111.24(15)$ & $\mathrm{N} 4^{\mathrm{i}}-\mathrm{LI}-\mathrm{N} 3^{\mathrm{i}}$ & $64.58(12)$ \\
\hline $\mathrm{C} 30-\mathrm{C} 25-\mathrm{C} 26$ & $110.27(15)$ & $\mathrm{N} 3-\mathrm{LI}-\mathrm{N} 3^{\mathrm{i}}$ & $111.00(17)$ \\
\hline N3-C25-H33 & 108.6 & $\mathrm{O}-\mathrm{LI}-\mathrm{C} 19^{\mathrm{i}}$ & $112.66(17)$ \\
\hline $\mathrm{C} 30-\mathrm{C} 25-\mathrm{H} 33$ & 108.6 & $\mathrm{~N} 4^{\mathrm{i}}-\mathrm{LI}-\mathrm{C} 19^{\mathrm{i}}$ & $32.96(8)$ \\
\hline $\mathrm{C} 26-\mathrm{C} 25-\mathrm{H} 33$ & 108.6 & $\mathrm{~N} 3-\mathrm{LI}-\mathrm{C} 19^{\mathrm{i}}$ & $129.55(18)$ \\
\hline $\mathrm{C} 27-\mathrm{C} 26-\mathrm{C} 25$ & $112.17(17)$ & $\mathrm{N} 3^{\mathrm{i}}-\mathrm{LI}-\mathrm{C} 19^{\mathrm{i}}$ & $32.85(7)$ \\
\hline $\mathrm{C} 27-\mathrm{C} 26-\mathrm{H} 34$ & 109.2 & $\mathrm{O}-\mathrm{LI}-\mathrm{LI}^{\mathrm{i}}$ & $131.6(3)$ \\
\hline $\mathrm{C} 25-\mathrm{C} 26-\mathrm{H} 34$ & 109.2 & $\mathrm{~N} 4^{\mathrm{i}}-\mathrm{LI}-\mathrm{LI}^{\mathrm{i}}$ & $94.2(2)$ \\
\hline $\mathrm{C} 27-\mathrm{C} 26-\mathrm{H} 35$ & 109.2 & $\mathrm{~N} 3-\mathrm{LI}-\mathrm{LI}^{\mathrm{i}}$ & $59.91(14)$ \\
\hline $\mathrm{C} 25-\mathrm{C} 26-\mathrm{H} 35$ & 109.2 & $\mathrm{~N} 3^{\mathrm{i}}-\mathrm{LI}-\mathrm{LI}^{\mathrm{i}}$ & $51.08(14)$ \\
\hline $\mathrm{H} 34-\mathrm{C} 26-\mathrm{H} 35$ & 107.9 & $\mathrm{C} 19^{\mathrm{i}}-\mathrm{LI}-\mathrm{LI}^{\mathrm{i}}$ & $75.64(17)$ \\
\hline $\mathrm{C} 28-\mathrm{C} 27-\mathrm{C} 26$ & $110.99(17)$ & $\mathrm{C} 40-\mathrm{O}-\mathrm{C} 37$ & $109.2(2)$ \\
\hline $\mathrm{C} 28-\mathrm{C} 27-\mathrm{H} 36$ & 109.4 & $\mathrm{C} 40-\mathrm{O}-\mathrm{LI}$ & $118.0(2)$ \\
\hline $\mathrm{C} 26-\mathrm{C} 27-\mathrm{H} 36$ & 109.4 & $\mathrm{C} 37-\mathrm{O}-\mathrm{LI}$ & $123.21(19)$ \\
\hline $\mathrm{C} 28-\mathrm{C} 27-\mathrm{H} 37$ & 109.4 & & \\
\hline $\mathrm{C} 3-\mathrm{C} 4-\mathrm{C} 5-\mathrm{C} 6$ & $108.2(2)$ & $\mathrm{C} 44-\mathrm{C} 42 \mathrm{~A}-\mathrm{C} 43-\mathrm{C} 41^{\mathrm{ii}}$ & $177.3(3)$ \\
\hline $\mathrm{C} 3-\mathrm{C} 4-\mathrm{C} 6-\mathrm{C} 5$ & $-107.4(2)$ & $\mathrm{N} 2-\mathrm{C} 1-\mathrm{N} 1-\mathrm{C} 7$ & $-174.14(16)$ \\
\hline $\mathrm{N} 1-\mathrm{C} 7-\mathrm{C} 8-\mathrm{C} 9$ & $177.97(19)$ & $\mathrm{C} 2-\mathrm{C} 1-\mathrm{N} 1-\mathrm{C} 7$ & $6.6(2)$ \\
\hline $\mathrm{C} 12-\mathrm{C} 7-\mathrm{C} 8-\mathrm{C} 9$ & $55.4(2)$ & $\mathrm{N} 2-\mathrm{C} 1-\mathrm{N} 1-\mathrm{AG}$ & $2.2(2)$ \\
\hline
\end{tabular}




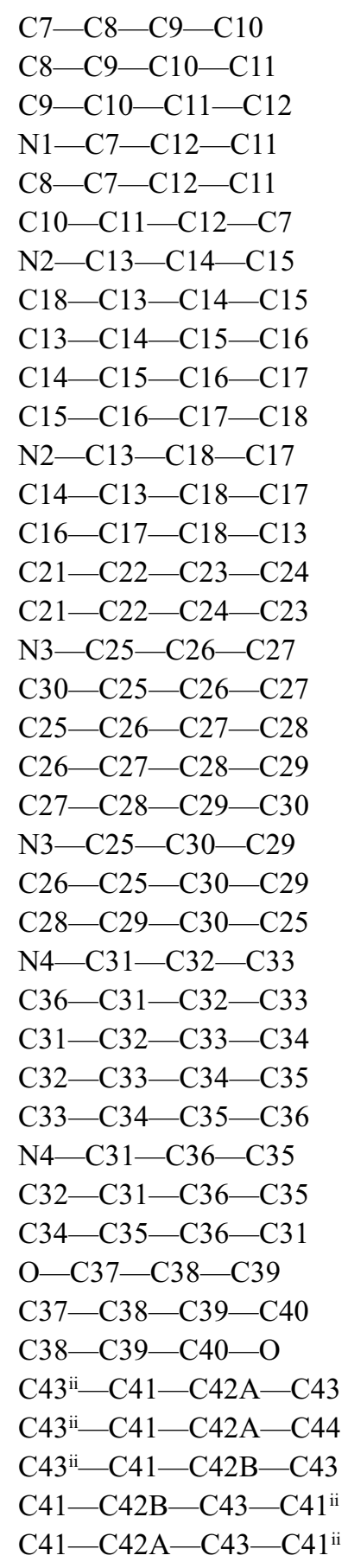

$-55.3(3)$
$54.4(3)$
$-54.5(3)$
$-178.41(16)$
$-55.5(2)$
$55.2(3)$
$179.88(16)$
$57.1(2)$
$-56.7(2)$
$54.8(2)$
$-54.2(2)$
$179.80(15)$
$-56.7(2)$
$55.6(2)$
$-107.76(19)$
$108.4(2)$
$175.81(16)$
$54.1(2)$
$-57.2(2)$
$56.8(2)$
$-55.1(2)$
$-174.89(16)$
$-52.1(2)$
$53.6(2)$
$-177.9(2)$
$-56.9(3)$
$56.0(4)$
$-54.5(4)$
$54.4(4)$
$178.0(2)$
$56.1(3)$
$-55.0(3)$
$26.4(4)$
$-32.0(4)$
$26.0(4)$
$0.8(4)$
$-177.2(3)$
$0.8(4)$
$-0.8(4)$
$-0.8(4)$

\begin{tabular}{|c|c|}
\hline $\mathrm{C} 2-\mathrm{C} 1-\mathrm{N} 1-\mathrm{AG}$ & $-177.07(12)$ \\
\hline $\mathrm{C} 8-\mathrm{C} 7-\mathrm{N} 1-\mathrm{C} 1$ & $96.9(2)$ \\
\hline $\mathrm{C} 12-\mathrm{C} 7-\mathrm{N} 1-\mathrm{C} 1$ & $-140.43(17)$ \\
\hline $\mathrm{C} 8-\mathrm{C} 7-\mathrm{N} 1-\mathrm{AG}$ & $-79.60(17)$ \\
\hline $\mathrm{C} 12-\mathrm{C} 7-\mathrm{N} 1-\mathrm{AG}$ & $43.06(18)$ \\
\hline $\mathrm{N} 1-\mathrm{C} 1-\mathrm{N} 2-\mathrm{C} 13$ & $-177.37(16)$ \\
\hline $\mathrm{C} 2-\mathrm{C} 1-\mathrm{N} 2-\mathrm{C} 13$ & $1.9(2)$ \\
\hline $\mathrm{N} 1-\mathrm{C} 1-\mathrm{N} 2-\mathrm{AG}^{\mathrm{iii}}$ & $-3.6(2)$ \\
\hline $\mathrm{C} 2-\mathrm{C} 1-\mathrm{N} 2-\mathrm{AG}^{\mathrm{iii}}$ & $175.62(12)$ \\
\hline $\mathrm{C} 18-\mathrm{C} 13-\mathrm{N} 2-\mathrm{C} 1$ & $-153.85(16)$ \\
\hline $\mathrm{C} 14-\mathrm{C} 13-\mathrm{N} 2-\mathrm{C} 1$ & $83.74(19)$ \\
\hline $\mathrm{C} 18-\mathrm{C} 13-\mathrm{N} 2-\mathrm{AG}^{\mathrm{iii}}$ & $32.10(18)$ \\
\hline $\mathrm{C} 14-\mathrm{C} 13-\mathrm{N} 2-\mathrm{AG}^{\mathrm{iii}}$ & $-90.30(16)$ \\
\hline N4-C19-N3-C25 & $172.85(17)$ \\
\hline $\mathrm{C} 20-\mathrm{C} 19-\mathrm{N} 3-\mathrm{C} 25$ & $-4.1(3)$ \\
\hline $\mathrm{LI}-\mathrm{C} 19-\mathrm{N} 3-\mathrm{C} 25$ & $153.26(19)$ \\
\hline N4-C19-N3-LI & $-37.3(3)$ \\
\hline $\mathrm{C} 20-\mathrm{C} 19-\mathrm{N} 3-\mathrm{LI}$ & $145.79(19)$ \\
\hline 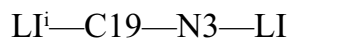 & $-56.9(2)$ \\
\hline $\mathrm{N} 4-\mathrm{C} 19-\mathrm{N} 3-\mathrm{LI}^{\mathrm{i}}$ & $19.59(19)$ \\
\hline $\mathrm{C} 20-\mathrm{C} 19-\mathrm{N} 3-\mathrm{LI}^{\mathrm{i}}$ & $-157.33(19)$ \\
\hline $\mathrm{C} 30-\mathrm{C} 25-\mathrm{N} 3-\mathrm{C} 19$ & $-140.43(17)$ \\
\hline $\mathrm{C} 26-\mathrm{C} 25-\mathrm{N} 3-\mathrm{C} 19$ & $97.41(19)$ \\
\hline $\mathrm{C} 30-\mathrm{C} 25-\mathrm{N} 3-\mathrm{LI}$ & $66.4(2)$ \\
\hline $\mathrm{C} 26-\mathrm{C} 25-\mathrm{N} 3-\mathrm{LI}$ & $-55.8(2)$ \\
\hline $\mathrm{C} 30-\mathrm{C} 25-\mathrm{N} 3-\mathrm{LI}^{\mathrm{i}}$ & $-18.6(3)$ \\
\hline $\mathrm{C} 26-\mathrm{C} 25-\mathrm{N} 3-\mathrm{LI}^{\mathrm{i}}$ & $-140.7(2)$ \\
\hline $\mathrm{N} 3-\mathrm{C} 19-\mathrm{N} 4-\mathrm{C} 31$ & $174.79(18)$ \\
\hline $\mathrm{C} 20-\mathrm{C} 19-\mathrm{N} 4-\mathrm{C} 31$ & $-8.3(3)$ \\
\hline $\mathrm{LI}-\mathrm{C} 19-\mathrm{N} 4-\mathrm{C} 31$ & $-163.3(2)$ \\
\hline $\mathrm{N} 3-\mathrm{C} 19-\mathrm{N} 4-\mathrm{LI}^{\mathrm{i}}$ & $-21.9(2)$ \\
\hline $\mathrm{C} 20-\mathrm{C} 19-\mathrm{N} 4-\mathrm{LI}^{\mathrm{i}}$ & $154.99(18)$ \\
\hline $\mathrm{C} 32-\mathrm{C} 31-\mathrm{N} 4-\mathrm{C} 19$ & $-90.0(2)$ \\
\hline $\mathrm{C} 36-\mathrm{C} 31-\mathrm{N} 4-\mathrm{C} 19$ & $148.8(2)$ \\
\hline $\mathrm{C} 32-\mathrm{C} 31-\mathrm{N} 4-\mathrm{LI}^{\mathrm{i}}$ & $119.8(3)$ \\
\hline $\mathrm{C} 36-\mathrm{C} 31-\mathrm{N} 4-\mathrm{LI}^{\mathrm{i}}$ & $-1.3(4)$ \\
\hline $\mathrm{C} 39-\mathrm{C} 40-\mathrm{O}-\mathrm{C} 37$ & $-10.2(4)$ \\
\hline $\mathrm{C} 39-\mathrm{C} 40-\mathrm{O}-\mathrm{LI}$ & $-157.6(3)$ \\
\hline $\mathrm{C} 38-\mathrm{C} 37-\mathrm{O}-\mathrm{C} 40$ & $-10.2(4)$ \\
\hline $\mathrm{C} 38-\mathrm{C} 37-\mathrm{O}-\mathrm{LI}$ & $135.2(3)$ \\
\hline
\end{tabular}

Symmetry codes: (i) $-x+2,-y+1,-z$; (ii) $-x+1,-y+2,-z$; (iii) $-x+2,-y+2,-z+1$. 